Boletín de la Sociedad Geológica Mexicana

VOLUMEN 64, NÚM. 3, 2012, P. 335-352

\title{
Petrografía y procedencia del miembro arenoso Galeana (Formación Taraises, Valanginiano - Hauteriviano temprano), Sierra Madre Oriental, NE México
}

\author{
Yam Zul Ernesto Ocampo-Díaz ${ }^{1, *}$, Martín Guerrero-Suastegui ${ }^{2}$, Uwe Jenchen ${ }^{3}$ \\ ${ }^{1}$ Área de Ciencias de la Tierra, Facultad de Ingeniería, Universidad Autónoma de San Luis Potosí, S.L.P., Av. Manuel Nava No. 8 , \\ Zona Universitaria, San Luis Potoís, México, CP 78290. \\ ${ }^{2}$ Unidad Académica de Ciencias de la Tierra, UAGro, Ex-Hacienda de San Juan Bautista, S/N, Taxco el Viejo, Guerrero, México. \\ ${ }^{3}$ Facultad de Ciencias de la Tierra, UANL, Carretera a Cerro Prieto Km. 8, Ex-Hacienda de Guadalupe, CP 67000, Linares, Nuevo \\ León, México. \\ *magonegro_2000@hotmail.com
}

\begin{abstract}
Resumen
Los sistemas extensionales se caracterizan por multifases de reactivación de sus fallas que propician la erosión y redistribución de los sedimentos dentro de la cuenca, influyendo incluso en la composición de las areniscas. El análisis de procedencia en secuencias sedimentarias depositadas dentro de este tipo de cuencas, permite documentar las etapas de evolución de estos ambientes, así como también la composición de los bloques del basamento que se encuentran aledaños o soterrados. El presente trabajo documenta por medio de un riguroso análisis petrográfico y un conteo de puntos en 58 muestras de areniscas del miembro arenoso Galeana (Formación Taraises; Cretácico Temprano), la composición y ubicación de las áreas fuentes que propiciaron su origen, además de la evolución petrotectónica de la porción sur de la Fosa de Monterrey durante el Cretácico Temprano. Petrográficamente, las areniscas del miembro arenoso Galeana pueden ser divididas en tres petrofacies y dos secuencias de procedencia que están estrechamente relacionadas con el ambiente sedimentario en que fueron depositadas. La petrofacies A "sedimentoclástica" corresponde a depósitos de facies mareales y representa la erosión de rocas preexistentes del pre-Cretácico Temprano, con una influencia moderada de rocas del basamento de composición granítica y/o granodiorítica y esquistosa. La petrofacies B "sedimento-plutonoclástica” corresponde a un cambio lateral de facies de la petrofacies A (norte-sur), que muestra un mayor índice de reciclamiento asociado al ambiente sedimentario de dunas subacuosas, cambios en la composición del área fuente, y reciclamiento interno (intraformacional o canibalismo). La petrofacies $\mathrm{C}$ marca el depósito de una segunda secuencia de procedencia representada por un cambio lateral de las petrofacies A y B con dirección Este-Oeste. Las areniscas representan la mayor exposición y erosión profunda de las rocas del basamento.

Las diferencias composicionales de los ensambles líticos sugieren un transporte sedimentario durante el Cretácico Temprano, desde el Archipiélago de Tamaulipas e Isla de Terán en el este hacia la Fosa de Monterrey, que propició la erosión de las rocas que hasta ese momento conformaban la cubierta sedimentaria y en menor grado de rocas plutónicas, volcánicas y metamórficas de grado bajo a medio. Dicha erosión dio paso al depósito de la primera secuencia de procedencia (petrofacies A y B). Por otra parte, el Hauteriviano temprano representa a la segunda secuencia de procedencia (petrofacies C), y al tiempo de mayor exhumación de bloques. Por consiguiente, se propone que durante el Valanginiano temprano-Hauteriviano medio, la Fosa de Monterrey estuvo expuesta a procesos extensivos relacionados con sistemas laterales de tipo strike-slip que propiciaron la exhumación de rocas que conforman el basamento.
\end{abstract}

Palabras clave: procedencia, petrografía sedimentaria, Sierra Madre Oriental, Formación Taraises, miembro arenoso Galeana, NE México. 


\begin{abstract}
Extensional systems are characterized by multiphase reactivation of faults that are conducive to erosion, redistribution of sediments within the basin and affect the composition of the sandstones. The provenance analysis of these sedimentary sequences documents the stages of evolution of these systems, as well as the composition of the buried basement blocks. This paper is based on a rigorous petrographical analysis and point counting in 58 samples of sandstones from the Galeana sandstone member, Taraises Formation (Early Cretaceous). It documents the composition and location of source areas that led to the origin of the sandstones, and the petrotectonic evolution of the southern region of the Monterrey Trough during the Early Cretaceous. Petrographically, the Galeana sandstone member can be divided into three petrofacies and two provenance sequences that are closely related to the sedimentary environment in which they were deposited. Sedimentoclastic petrofacies A corresponds to tidal facies deposits and represents the erosion of preexisting rocks from the pre-Early Cretaceous, with a moderate influence of basement rocks of granitic and/or granodioritic and schistose composition. Sedimentary-plutonoclastic petrofacies B corresponds to a shift in lateral facies from petrofacies A (north-south). It shows a higher recycling index associated with the subaqueous dune sedimentary environment, changes in the source area composition, and internal recycling (intraformational or cannibalism). Petrofacies $C$ marks the deposition of a second provenance sequence, represented by an east-west lateral change in petrofacies $A$ and $B$. The sandstones represent the greatest exposure and erosion of deep basement rocks.

The compositional differences between the lithic assemblages suggest that a sedimentary transport event occurred during the Early Cretaceous, from the Tamaulipas Arch and Terán Island in the east to the Monterrey Trough, favoring the erosion of the sedimentary cover, and to a minor degree plutonic, volcanic and low to medium-grade metamorphic rocks. Such erosion led to the deposition of the first provenance sequence (petrofacies $A$ and B). On the other hand, the early Hauterivian represents a second provenance sequence (petrofacies $C$ ), and the major period of block exhumation.

Therefore, this paper proposes that during the early Valanginian-middle Hauterivian, the Monterrey Trough was exposed to extensional processes related to strike-slip systems that produced the exhumation of basement rocks.
\end{abstract}

Keywords: provenance, sedimentary petrography, Sierra Madre Oriental, Taraises Formation, Galeana sandstone member, NE Mexico.

\section{Introducción}

Los sistemas extensionales se caracterizan por multifases de reactivación de sus fallas, propiciando la erosión, la redistribución de los sedimentos dentro de la cuenca y la composición de las areniscas (Arribas et al., 2003; Garzanti et al., 2003; González-Acebrón et al., 2007; Marsaglia et al., 2007). La primera etapa de los rift y cuencas pull-apart inicia con la erosión de las rocas de cobertura preexistentes $\mathrm{y}$ en menor grado de las rocas que conforman el basamento. Las etapas subsecuentes involucran la erosión profunda del basamento y el reciclamiento interno durante el depósito. $\mathrm{La}$ procedencia dentro de este tipo de secuencias documenta las diferentes etapas de evolución de estos sistemas, así como también la composición de los bloques del basamento, que serán enterrados por la cubierta sedimentaria conforme evoluciona un rift a una etapa de subsidencia contínua.

La actividad tectónica es considerada el factor principal que controla la sedimentación dentro de cuencas de rift o sistemas pull-apart (Prosser, 1993). Las cuencas asociadas a sistemas pull-apart y sistemas de rift generalmente son rellenadas por el material erosionado de los altos que la bordean, siendo esto el resultado de la extensión litosférica y levantamiento de bloques asociado con reactivación de fallas. Por consiguiente, la composición de los sedimentos clásticos depende tanto de la naturaleza de los bloques de basamento como de la actividad tectónica y de las condiciones climáticas que controlan el régimen de reanudación sedimentaria (Johnson, 1993). Ingersoll (1983) y Arribas et al. (2003) sugieren que la composición de areniscas y petrofacies son sensitivas a los cambios dentro de las áreas fuentes, así como también a su evolución a través del tiempo, por consiguiente pueden ser empleadas para documentar o definir secuencias deposicionales.

La petrografía sedimentaria de rocas clásticas es útil para realizar reconstrucciones paleogeográficas y tectónicas, permitiendo además determinar el área fuente de rocas sedimentarias clásticas antiguas (Blatt et al., 1972; Dickinson, 1970; Pettijohn et al., 1972; Dickinson et al., 1983). La composición final de las rocas siliciclásticas se encuentra influenciada por muchos factores como: i) composición del área fuente; ii) distancia de transporte; iii) relieve; iv) morfología de la cuenca; v) clima y vi) diagénesis (Blatt, 1967, Suttner et al., 1981, Ricci-Lucchi, 1985; Johnson, 1993; Cox y Lowe, 1995). Diversos autores sugieren que la composición de las rocas siliciclásticas registra el ambiente tectónico en que fueron depositadas (e.g., Dickinson y Suczek, 1979; Dickinson et al., 1983; Mack, 1984; Valloni, 1985). Los principales trabajos sobre la composición y ambiente tectónico de las rocas siliciclásticas han documentado ampliamente la evolución de cuencas de tipo foreland (entre otros, Hiscott, 1978; DeCelles y Hertel, 1989; Critelli y Le Pera, 1995; Critelli, 1999), siendo pocos los estudios que documentan las 
características petrográficas y diagenéticas de cuencas dentro un sistema extensional (Rift o pull-apart; Zuffa et al., 1980; Marsaglia, 1991; Garzanti et al., 2001; Critelli et al., 2002, Arribas et al., 2003; Garzanti et al., 2003; GonzálezAcebrón et al., 2007).

El norte de México, se encuentra dividido en cuatro cuencas sedimentarias que inician como cuencas de tipo rift intra-placa y posteriormente evolucionan a cuencas de tipo strike-slip (Cuenca de Bisbee, Fosa de Chihuahua, Cuenca de Sabinas y Fosa de Monterrey; Tardy et al., 1989; Ocampo-Díaz, 2011; Figura 1), que recibieron su máximo aporte de sedimentos clásticos durante el Jurásico TardíoCretácico Temprano (Michalzik, 1988, 1991; Goldhammer, 1999; Pindell y Kennan, 2002; Ocampo-Díaz, 2007). Dentro de la Fosa de Monterrey aflora una secuencia clástica que ha sido denominada miembro mrenoso Galeana, de la Formación Taraises, del Cretácico Temprano, del cual se ha postulado que procede de la porción sureste del Bloque de Coahuila.
El presente trabajo documenta: (1) las características petrográficas del miembro arenoso Galeana (ValanginianoHauteriviano temprano), depositado en ambientes costeros y deltaicos en la porción sur de la Fosa de Monterrey; (2) las diferencias composicionales y litológicas de las rocas fuente que le dieron origen y que actualmente son cubiertas por la columna estratigráfica del noreste de México.

\section{Geología regional}

El área de estudio se localiza dentro la Fosa de Monterrey cuyo relleno sedimentario forma parte de la cubierta sedimentaria del terreno tectonoestratigráfico Sierra Madre (Campa y Coney, 1983). El basamento del Terreno Sierra Madre está conformado por rocas metamórficas de edad precámbrica ( 1 Ga, Gneiss Novillo), que están en contacto tectónico con esquistos pelíticos y psamíticos con lentes de serpentinitas, metagabros, metabasaltos y



Figura 1. Imagen superior: delimitación de las tres cuencas en el noreste de México (Tomado de Ocampo-Díaz et al., 2008). Imagen principal: Mapa geológico regional del noreste de México (Curvatura de Monterrey; Tomado de Michalzik, 1986). En el recuadro se marca el área de estudio. 
metapedernal del Esquisto Granjeno-Aramberri (CarrilloBravo, 1961; Campa y Coney, 1983; Keppie, 2004; Dowe et al., 2005). Tanto el Gneiss Novillo, como el Esquisto Granjeno presentan como cubierta sedimentaria paleozoica, secuencias conglomeráticas, rocas carbonatadas, tobas riolíticas, lutitas y areniscas del Silúrico-Devónico (Carrillo-Bravo, 1961; Gursky y Michalzik, 1989). Las rocas precámbricas y paleozoicas fueron intrusionadas por granitos y granodioritas de edad Pérmico-Triásico documentadas en el noreste de México como Isla La Mula y Archipiélago de Tamaulipas (McKee et al., 1990; Jones et al., 1995; Torres et al., 1999; Pindell y Kennan, 2002).

Durante el Triásico Medio-Jurásico Medio, en la margen paleo-pacífica de México, se depositaron diferentes secuencias siliciclásticas y volcánicas de composición intermedia-félsica y sedimentos volcaniclásticos relacionados con arcos continentales emplazados en la porción suroeste de Norte América (Grajales-Nishimura et al., 1992; Jones et al., 1995; Bartolini et al., 2001; Barboza-Gudiño et al., 1998, 1999, 2008), denominadas como Formación Nazas (Zacatecas, San Luís Potosí y Durango), Serie Pre-Huizachal (Nuevo León; Meiburg et al., 1987) y Formación La Boca (Tamaulipas; Mixon et al., 1959). Las edades U-Pb de estas rocas varían de 185 Ma hasta 195 Ma (Fastovsky et al., 2005; Barboza-Gudiño et al., 2008; Rubio-Cisneros y Lawton, 2011).

2.1. Geología Regional y Estratigrafía del Jurásico Medio al Cretácico Temprano

Durante el Jurásico Medio-Cretácico Temprano, debido a un régimen de subducción oblicua situado en la margen paleo-pacífica, se desarrolló fallamiento lateral izquierdo y sistemas transtensivos, generando cuencas intra-cratónicas, pull-apart y strike-slip denominadas: Sistema de Cuencas strike-slip McCoy-Sabinas (Tardy et al., 1989; Bassett y Busby, 2005; Mickus et al., 2009; Ocampo-Díaz, 2011). Entre tanto en la margen Atlántica, se iniciaba la apertura del Golfo de México (Michalzik, 1988; Fillon, 2007; Ocampo-Díaz, 2007), favoreciendo que se depositaran en las inmediaciones de los altos de basamentos secuencias carbonatadas y siliciclásticas en ambientes continentales y costeros (formaciones La Gloria y Minas Viejas; Buffler y Sawyer, 1985; Michalzik, 1986 y 1988; Salvador, 1991). Estos depósitos cambian verticalmente a facies lodosas de rampa externa y playa de la Formación Zuloaga (Michalzik, 1988, Goldhammer, 1999). La Formación Olvido sobreyace de manera concordante a la Formación Zuloaga y se caracteriza por depósitos evaporíticos, calcáreos y capas rojas (Michalzik, 1988; Götte, 1988). El Jurásico Tardío se conforma por depósitos marinos marginales (deltas y línea de costa) que cambian lateralmente a depósitos de plataforma abierta de la Formación La Casita (Michalzik, 1988; Michalzik y Shumman, 1994; Figuras 1 y 2). En el Cretácico Temprano se depositaron secuencias carbonatadas en ambientes de plataforma abierta, dentro de un régimen de subsidencia uniforme de la Formación Taraises (Michalzik, 1986, 1988), y también rocas clásticas dentro de sistemas costeros del miembro arenoso Galeana (Díaz-González, 1953; Michalzik, 1988; Ocampo-Díaz, 2007; Ocampo-Díaz et al., 2008).

\section{Estratigrafía y sedimentología del miembro arenoso Galeana}

\subsection{Estratigrafía}

Díaz-González (1953) fue el primero en reportar las areniscas del miembro arenoso Galeana, asignándole una edad Valanginiano temprano-Hauteriviano temprano, por la presencia de amonites del género Olcostephanus del grupo O. astieri-d'Orbiny, muestreados en la parte final del perfil situado en el Cerro de Labradores, a $1.5 \mathrm{~km}$. al este del poblado de Galeana, Nuevo León; además, sobreyace a la Formación Taraises que contiene amonites de los géneros Berriasella (?), Apiticeras, Neocomites sp., Thurmannites y Acanthodiscu (?), que permiten situarla dentro del Berriasiano-Valanginiano temprano (Ccf. Imlay, 1938). Michalzik (1988) realiza trabajos sedimentológicos detallados y cartografía de las formaciones que subyacen al miembro arenoso Galeana, que está constituido por intercalaciones de lutitas y areniscas. Las areniscas son de grano fino a grueso, de color gris claro que intermperizan a café ocre. El espesor total reportado varía de 130 a 450 m (Figura 3), en las áreas de Galeana, Potrero Prieto y Rayones ( $c f$., Ocampo-Díaz, 2007; Ocampo-Díaz et al., 2008), acuñándose hacia el norte y sur del área de estudio, donde solo afloran carbonatos de la Formación Taraises.

El miembro arenoso Galeana sobreyace de manera concordante al miembro inferior de la Formación Taraises en las áreas de Potrero Prieto y Rayones, y por discordancia angular en el área de Galeana; y subyace de manera transicional al miembro superior de la Formación Taraises. Este miembro es correlacionable con las formaciones Las Vigas, Capulín, La Carbonera y la Arcosa Patula (DíazGonzález, 1953; Michalzik, 1988; Eguiluz de Antuñano, 1990).

\subsection{Sedimentología}

De acuerdo con Ocampo-Díaz (2007) y Ocampo-Díaz et al. (2008), las areniscas del miembro arenoso Galeana presentan siete asociaciones de facies que se interpretan como depósitos prodelta y frente deltaico influenciado por mareas, de los cuales se identificaron los subambientes de planicie mareal, canales mareales, planicie de lodos y depósitos de dunas subacuosas. Los mismos autores proponen la siguientes características sedimentológicas de estas asociaciones de facies, siendo: (1) secuencias estrato-crecientes y estrato-decrecientes; (2) abundante gradación normal, estratificación lenticular, estratificación 


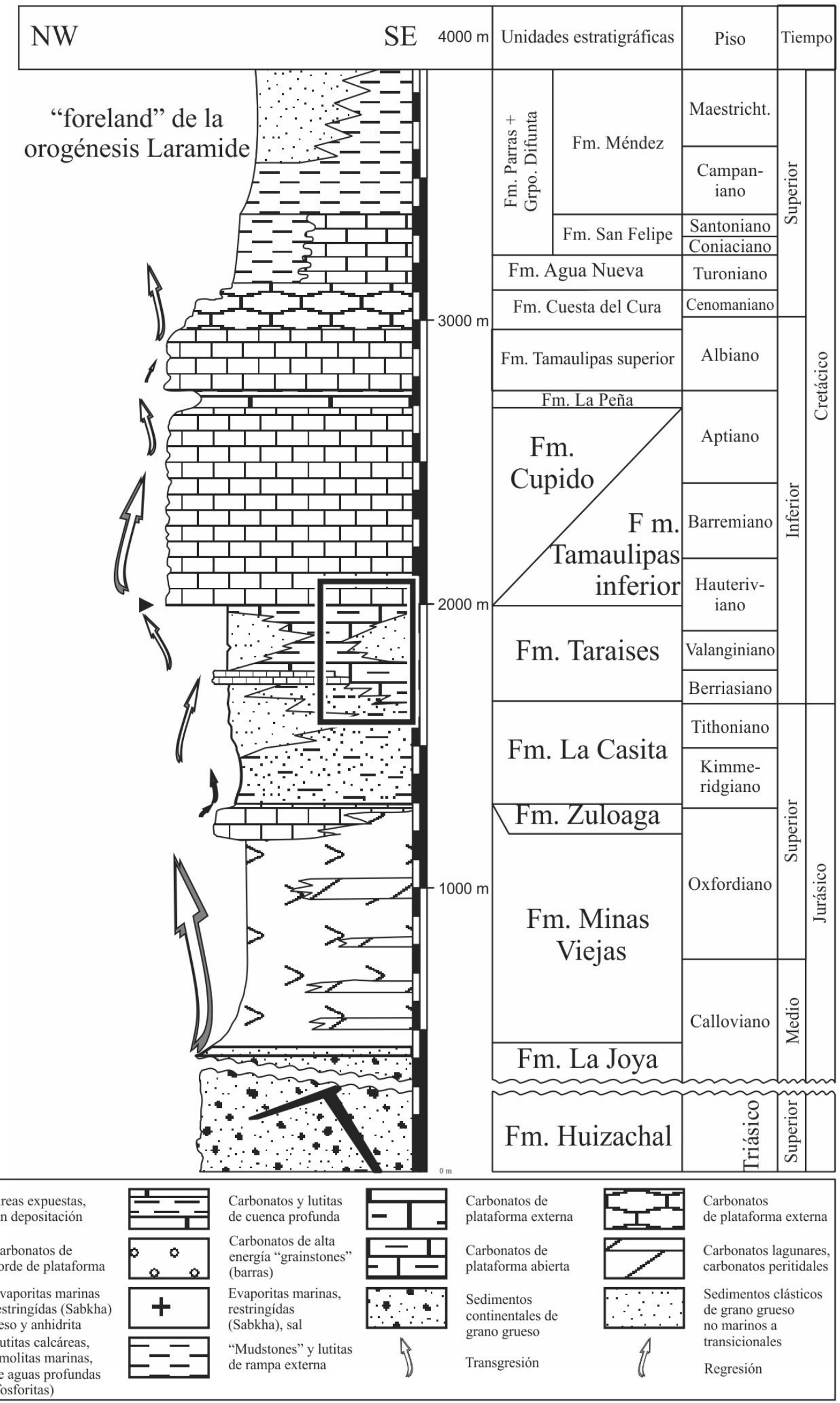

Figura 2. Columna estratigráfica del noreste de México (Tomada de Michalzik, 1988 y Ocampo-Díaz et al., 2008) dentro del recuadro negro se resaltan las areniscas estudiadas. 


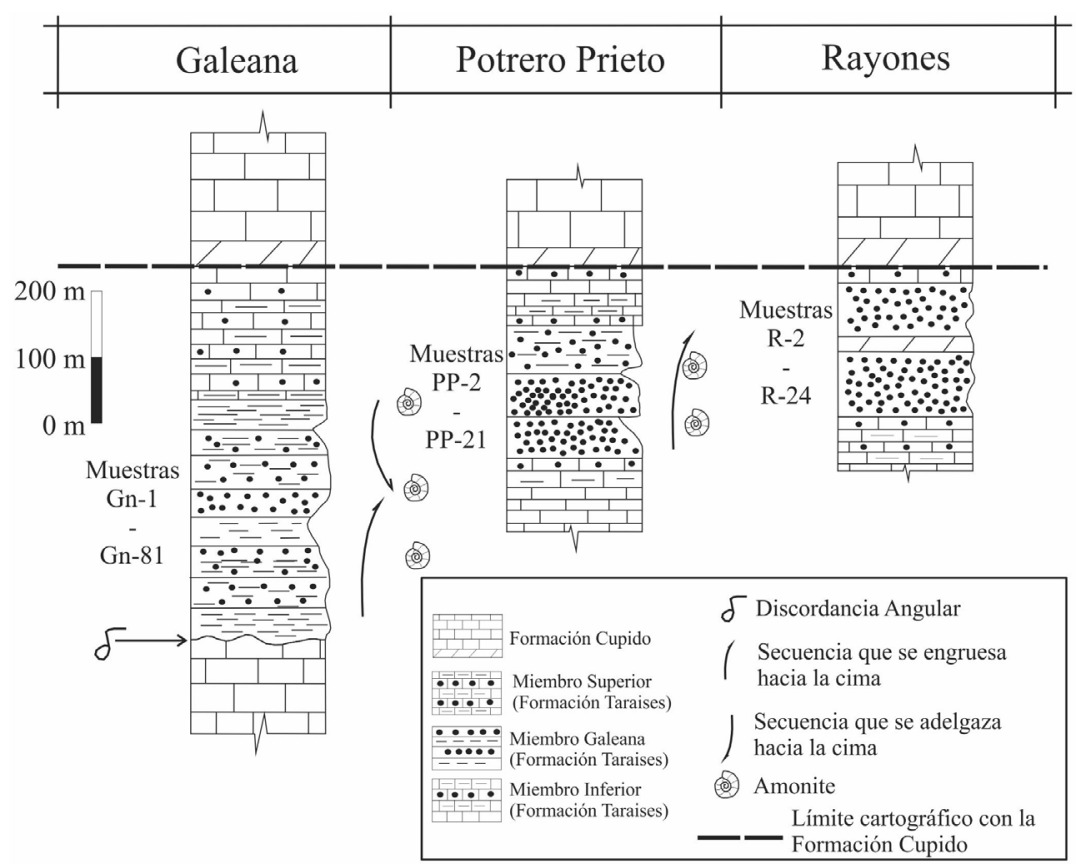

Figura 3. Columnas estratigráficas de las tres áreas de estudio, mostrando la ubicación de las muestras (tomada de Ocampo-Díaz, 2007 y Ocampo-Díaz et al., 2008).

cruzada de tipo herringbone, estratificación de tipo flaser y estratificación cruzada de tipo tangencial, generada por la migración de las dunas; (3) fósiles traza reconocidos como Tallasinoides, Skolithos, Diplocaterium y Rhizocorallium; (4) canales mareales con secuencias estrato-decrecientes, gradación normal, laminaciones cruzadas de tipo planar y rara vez tangencial, y lodos acoplados dobles. Estas características permiten interpretar a las areniscas del miembro arenoso Galeana como depósitos deltaicos influenciados por mareas con subambientes de planicie mareal (área de Potrero Prieto), sistema deltaico influenciado por mareas y depósitos sublitorales (área de Galeana) y depósitos de dunas subacuosas (área de Rayones). Los depósitos de planicie mareal progradan a depósitos del sistema deltaico (este-oeste) y cambian lateralmente a depósitos de dunas subacuosas (norte-sur; Ocampo-Díaz et al., 2008; Figura 4).

\section{Metodología}

El análisis petrográfico se realizó sobre 140 muestras de areniscas de grano medio a fino, de las áreas de Potrero Prieto, Rayones y Galena (Figura 4). El análisis modal se efectuó en 58 muestras seleccionadas de areniscas de grano medio (Figura 3). Las láminas delgadas fueron atacadas químicamente con HF y teñidas con una solución hiperconcentrada de cobaltonitrito de sodio y cloruro de bario, para diferenciación rápida de los feldespatos potásicos y plagioclasas, de acuerdo con la metodología de Marsaglia y Tazaki (1992). El análisis petrográfico cuantitativo se elaboró por medio de un conteo de 400 a 500 granos libres de matriz por lámina delgada, utilizando el método de GazziDickinson (Ingersoll et al., 1984). Los tipos de cuarzos fueron clasificados de acuerdo a Basu (1976), Basu et al. (1975), Blatt y Christie (1963) y Young (1976). Los líticos volcánicos se diferenciaron bajo los criterios de Dickinson (1970) y Marsaglia et al. (1992), mientras que los granos metamórficos fueron clasificados de acuerdo a Garzanti y Vezzoli (2003), quienes emplean subíndices para indicar el grado metamórfico de la roca madre. Los parámetros petrográficos empleados en los diagramas ternarios son mostrados en la Tabla 1 y se obtuvieron de acuerdo a los criterios de McBride (1963; QFL), Dickinson (1985), Dickinson y Suczek (1979; QmFLt; QmPK). Además de las metodologías de Arribas et al. (1990), Critelli e Ingersoll (1994) y Arribas et al. (2003), se tomaron los parámetros que conforman los ensambles granosos (Rg), sedimentarios (Rs) y metámorficos (Rm). La base de datos del conteo de puntos de las 58 muestras es mostrada en la Tabla 2 y los parámetros estandarizados y sus medias aritméticas y desviaciones estándar son mostrados en las Tablas 3 y 4.

\section{Resultados}

Las areniscas del miembro arenoso Galeana muestran cambios significativos en sus modos detríticos y en su posición dentro de los diagramas empleados, por lo que han sido divididas en tres petrofacies distintas que coinciden con las tres divisiones litoestratigráficas propuestas por Ocampo-Díaz (2007; Tablas 2-4). En total, se reconocieron 


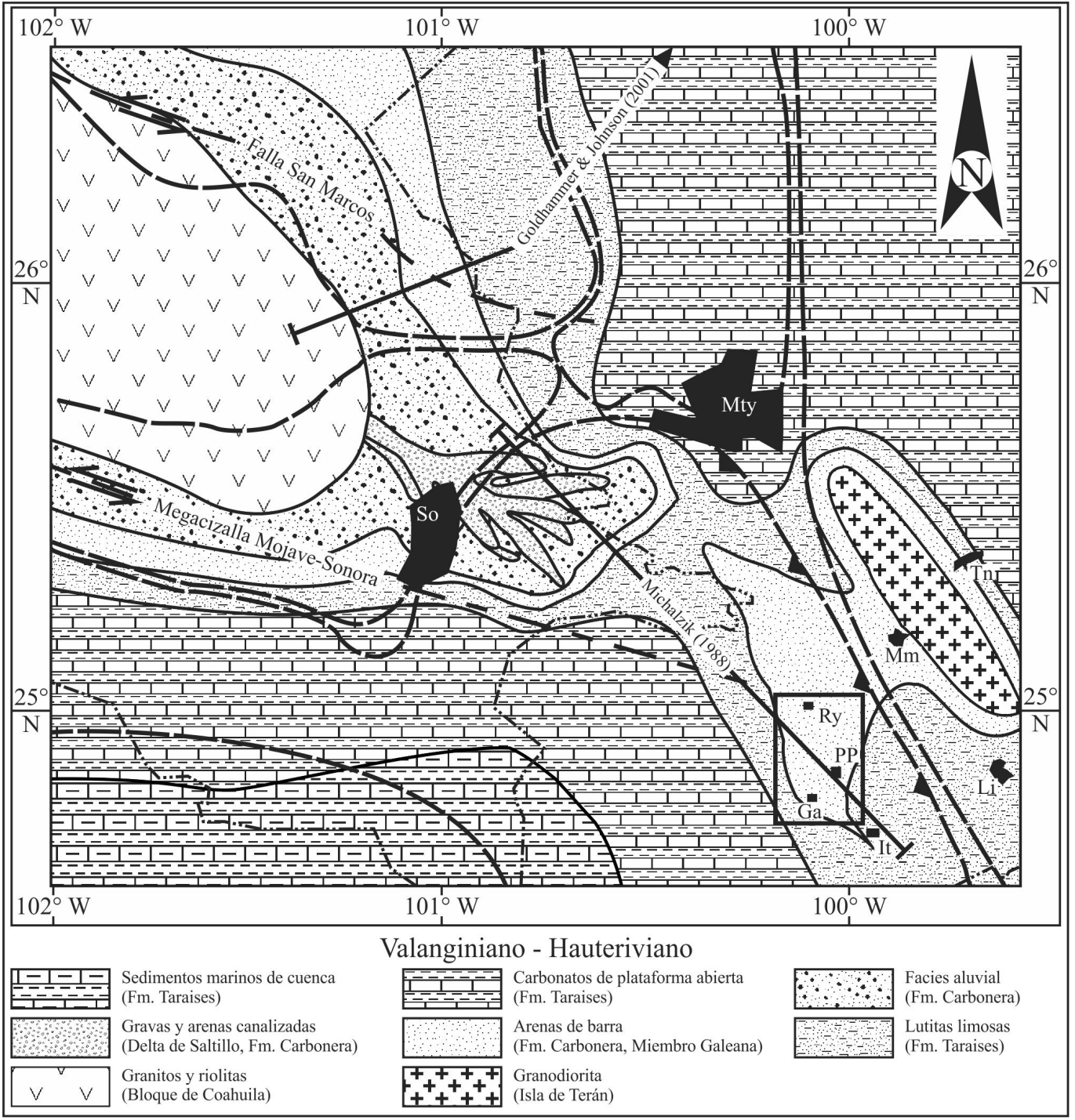

Figura 4. Reconstrucción paleogeográfica del NE de México para el Valanginiano - Hauteriviano: Sedimentación del miembro arenoso Galena, Formación Taraises (compilación de las paleoreconstrucciones de: Eguiluz de Antuñano, 1990, 2001; Goldhammer y Johnson, 2001; Jenchen, 2007a, b; y OcampoDíaz et al., 2008).

24 clases petrográficas, de las cuales 22 corresponden a granos y 2 representan la matriz y cemento (Tabla 2).

\subsection{Petrofacies A: Área de Potrero Prieto "Sedimentoclástica"}

Las areniscas de esta petrofacies son arcosas líticas que van de moderadas a bien clasificadas (Figura 5; $\mathrm{Q}_{59} \mathrm{~F}_{28} \mathrm{~L}_{13}$; McBride, 1963). La población más abundante está constituida por cuarzos monocristalinos subredondeados a redondeados (Figura 6A), con extinción no-ondulante $>$ ondulante. El cuarzo policristalino con más de tres cristales $(\mathrm{Qm}>3)$ es poco frecuente, mostrando textura tectónica caracterizada por contactos suturados y extinción individual grano a grano. Los granos de feldespatos son subangulosos a subredondeados, siendo más abundantes las plagioclasas con maclado polisintético que los feldespatos potásicos con maclado de tipo carlsbad ( $\mathrm{P} / \mathrm{F} \sim 1.35$; Tablas 3 y 4$)$. Los fragmentos líticos son subangulosos a subredondenados, siendo en orden de abundancia: fragmentos de areniscas de grano fino, fragmentos de lutitas, líticos carbonatados con texturas micríticas y esparíticas, abundante chert, líticos volcánicos con textura felsítica, (Figura 6A y 6B), líticos metamórficos metapsamíticos/felsíticos y escasos líticos metapsamíticos/felsíticos 3 y escasos fragmentos líticos plutónicos con texturas faneríticas de granodioritas o granitos. Los minerales accesorios incluyen circón, turmalina, moscovita y minerales opacos no identificados, además de fragmentos fósiles.

El contenido de matriz es $<13 \%$ siendo principalmente epimatriz y protomatriz (c.f., Dickinson, 1985), variando en un porcentaje de 7-13\%. El cemento es calcita cuyo 
porcentaje es menor del $7 \%$, en ocasiones en hábito poikilotípico, siendo común que se presente en agregados de pequeños cristales reemplazando al feldespato y en ocasiones bordeando al cuarzo.

Tabla 1. Parámetros recalculados empleados en los diagramas ternarios de acuerdo a McBride (1963) Dickinson et al. (1983), Dickinson y Suczek (1979), Arribas et al. (1990, 2003) y Critelli y Le Pera (1995).

\begin{tabular}{|c|c|}
\hline Diagrama Ternario & Parámetros \\
\hline \multirow{4}{*}{ QFR } & $\mathbf{Q}=\mathrm{Qm}+\mathrm{Qp}<3+\mathrm{Qp}>3+\mathrm{Qlm}+$ \\
\hline & $\mathrm{Qlp}+\mathrm{Qlv}+\mathrm{Q} 1 \mathrm{~s}+\mathrm{Qp}+\mathrm{Ch}$ \\
\hline & $\mathbf{F}=\mathrm{Fk}+\mathrm{Flv}+\mathrm{Pg}+\mathrm{Plv}$ \\
\hline & $\mathbf{R}=\mathrm{Lm}+\mathrm{Lv}+\mathrm{Ls}+\mathrm{Lsa}+$ \\
\hline \multirow{5}{*}{ QmFLt } & Lslu+Lsc+Lvf \\
\hline & $\mathbf{Q m}=\mathrm{Qm}+\mathrm{Qpl}+\mathrm{Qlp}+\mathrm{Qlv}+$ \\
\hline & Qls+Qp \\
\hline & $\mathbf{F}=\mathrm{Fk}+\mathrm{Flv}+\mathrm{Pg}+\mathrm{Plv}$ \\
\hline & $\mathbf{L t}=\mathbf{L m}+\mathrm{Lv}+\mathrm{Ls}+\mathrm{Lsa}+\mathrm{Lslu}+$ \\
\hline \multirow{5}{*}{ QmPK } & $\mathrm{Lsc}+\mathrm{Lvf}+\mathrm{Ch}$ \\
\hline & $\mathbf{Q m}=\mathrm{Qm}+\mathrm{Qpl}+\mathrm{Qlp}+\mathrm{Qlv}+$ \\
\hline & $\mathrm{Qls}+\mathrm{Qp}$ \\
\hline & $\mathbf{P}=\mathrm{Pg}+\mathrm{Plv}$ \\
\hline & $\mathbf{K}=\mathrm{Fk}+\mathrm{Flv}$ \\
\hline \multirow{3}{*}{ RgRsRm } & $\mathbf{R g}=$ Flv + Plv + LvLvf \\
\hline & $\mathbf{R s}=\mathrm{Ls}+\mathrm{Lslu}+\mathrm{Lsa}+\mathrm{Lsc}$ \\
\hline & $\mathbf{R m}=\mathrm{Lm}$ \\
\hline
\end{tabular}

5.2. Petrofacies B: Área Rayones "Sedimentoplutonoclástica"

La petrofacies B esta constituida por subarcosas líticas $\left(\mathrm{Q}_{63} \mathrm{~F}_{24} \mathrm{~L}_{13}\right.$; Figura 5), correspondiendo al área de Rayones. El cuarzo monocristalino con extinción no-ondulante es el más abundante de la fracción monomineral, generalmente es subanguloso a subredondeado. Dentro de esta petrofacies se presentan cuarzos de tipo volcánico con golfos de corrosión (Figura 6; Tablas 2-4). Los feldespatos alcalinos son subangulosos-subredondeados, con maclado tipo carlsbad, parcialmente reemplazados por caolinita. La plagioclasa, es principalmente de composición albita (Figura 6D), con maclado polisintético y combinado, en relaciones promedio entre plagioclasas contra feldespatos potásicos de $\mathrm{P} / \mathrm{K} \sim 1.99$. Los fragmentos líticos son subredondenados a redondenados, constituidos, en orden de abundancia, por areniscas de grano fino a medio (Figura 6E), fragmentos líticos volcánicos con texturas microlíticas y en menor proporción fragmentos de rocas carbonatadas con texturas micríticas y esparíticas.

Los minerales accesorios son principalmente biotita,

Tabla 2. Base de datos petrográfica obtenida del conteo de puntos en las areniscas del miembro arenoso Galeana

\begin{tabular}{|c|c|c|c|c|c|c|c|c|c|c|c|c|c|c|c|c|c|}
\hline & & Muestra & $\begin{array}{c}\text { Gn- } \\
1\end{array}$ & $\begin{array}{l}\text { Gn- } \\
4\end{array}$ & $\begin{array}{l}\text { Gn- } \\
6 \\
\end{array}$ & $\begin{array}{l}\text { Gn- } \\
9\end{array}$ & $\begin{array}{l}\text { Gn- } \\
10\end{array}$ & $\begin{array}{l}\text { Gn- } \\
11\end{array}$ & $\begin{array}{l}\text { Gn- } \\
21\end{array}$ & $\begin{array}{l}\text { Gn- } \\
24\end{array}$ & $\begin{array}{l}\text { Gn- } \\
27\end{array}$ & $\begin{array}{l}\text { Gn- } \\
33\end{array}$ & $\begin{array}{l}\text { Gn- } \\
39\end{array}$ & $\begin{array}{l}\text { Gn- } \\
40\end{array}$ & $\begin{array}{l}\text { Gn- } \\
46\end{array}$ & $\begin{array}{l}\text { Gn- } \\
46\end{array}$ & $\begin{array}{l}\text { Gn- } \\
48 \\
\end{array}$ \\
\hline \multirow[t]{9}{*}{$\mathbf{Q}$} & Qm & $\begin{array}{l}\text { Cuarzo monocristalino } \\
\text { con undolosidad }<5^{\circ} \mathrm{y}>5^{\circ}\end{array}$ & 266 & 260 & 253 & 210 & 245 & 235 & 245 & 272 & 260 & 275 & 265 & 257 & 250 & 245 & 260 \\
\hline & Qp2-3 & $\begin{array}{l}\text { Cuarzo policristalino con } \\
2-3 \text { cristales }\end{array}$ & 10 & 11 & 16 & 34 & 6 & 13 & 10 & 16 & 12 & 11 & 8 & 12 & 8 & 10 & 15 \\
\hline & $Q p>3$ & $\begin{array}{l}\text { Cuarzo policristalino } \\
\text { con }>3 \text { cristales }\end{array}$ & 28 & 42 & 48 & 20 & 16 & 18 & 30 & 27 & 26 & 30 & 20 & 31 & 32 & 30 & 10 \\
\hline & QIm & $\begin{array}{l}\text { Cuarzo dentro de un } \\
\text { lítico metamórfico }\end{array}$ & 5 & 0 & 0 & 7 & 0 & 2 & 8 & 2 & 0 & 1 & 0 & 5 & 0 & 10 & 2 \\
\hline & Qlp & $\begin{array}{l}\text { Cuarzo dentro de un } \\
\text { lítico plutónico }\end{array}$ & 7 & 8 & 10 & 6 & 3 & 0 & 5 & 3 & 10 & 8 & 3 & 4 & 12 & 8 & 0 \\
\hline & Qlv & $\begin{array}{l}\text { Cuarzo dentro de un } \\
\text { lítico volcánico }\end{array}$ & 0 & 0 & 0 & 4 & 5 & 2 & 0 & 0 & 0 & 7 & 0 & 2 & 0 & 1 & 2 \\
\hline & QIs & $\begin{array}{l}\text { Cuarzo dentro de un } \\
\text { lítico sedimentario }\end{array}$ & 0 & 0 & 0 & 0 & 0 & 4 & 0 & 0 & 0 & 0 & 0 & 1 & 0 & 0 & 0 \\
\hline & Qlp & $\begin{array}{l}\text { Cuarzo dentro de un } \\
\text { lítico plutónico }\end{array}$ & 0 & 0 & 2 & 0 & 0 & 0 & 0 & 0 & 0 & 0 & 2 & 0 & 0 & 0 & 0 \\
\hline & Ch & Chert & 8 & 11 & 12 & 11 & 0 & 10 & 12 & 10 & 6 & 9 & 10 & 6 & 7 & 10 & 11 \\
\hline \multirow[t]{3}{*}{$\mathbf{F}$} & Fs & $\begin{array}{l}\text { Feldespato Potásico en } \\
\text { un solo cristal }\end{array}$ & 52 & 40 & 42 & 50 & 86 & 42 & 55 & 55 & 64 & 65 & 70 & 58 & 89 & 68 & 55 \\
\hline & Flv & Feldespato potásico & & & & & & & & & & & & & & & \\
\hline & & $\begin{array}{l}\text { Dentro un fragmento de } \\
\text { roca volcánica }\end{array}$ & 3 & 5 & 1 & 1 & 0 & 2 & 4 & 0 & 2 & 1 & 3 & 3 & 1 & 4 & 0 \\
\hline \multirow[t]{2}{*}{$\mathbf{P}$} & Pg & $\begin{array}{l}\text { Plagioclasa en un solo } \\
\text { cristal }\end{array}$ & 73 & 100 & 102 & 75 & 108 & 88 & 77 & 75 & 94 & 70 & 95 & 56 & 92 & 100 & 80 \\
\hline & Plv & $\begin{array}{l}\text { Plagioclasa dentro de un } \\
\text { fragmento de roca } \\
\text { volcánica }\end{array}$ & 6 & 0 & 3 & 2 & 0 & 5 & 3 & 3 & 4 & 2 & 5 & 4 & 3 & 3 & 1 \\
\hline \multirow[t]{12}{*}{$\mathbf{L}$} & $\mathbf{L m}$ & Lítico metamórfico & 0 & 4 & 1 & 3 & 0 & 1 & 1 & 2 & 9 & 2 & 1 & 4 & 0 & 0 & 0 \\
\hline & $\mathbf{L v}$ & Lítico Volcánico & 0 & 1 & 5 & 4 & 0 & 3 & 0 & 0 & 5 & 0 & 0 & 0 & 0 & 0 & 0 \\
\hline & Ls & Lítico sedimentario & 0 & 0 & 2 & 27 & 0 & 25 & 0 & 0 & 0 & 6 & 0 & 32 & 0 & 0 & 21 \\
\hline & Lsa & $\begin{array}{l}\text { Lítico sedimentario de } \\
\text { areniscas }\end{array}$ & 40 & 28 & 39 & 0 & 31 & 0 & 52 & 34 & 38 & 36 & 25 & 0 & 30 & 37 & 0 \\
\hline & Lslu & $\begin{array}{l}\text { Lítico Sedimentario } \\
\text { de lutita }\end{array}$ & 0 & 0 & 0 & 0 & 0 & 0 & 0 & 0 & 0 & 0 & 0 & 0 & 0 & 0 & 0 \\
\hline & Lsc & $\begin{array}{l}\text { Lítico Sedimentario } \\
\text { carbonatado }\end{array}$ & 4 & 0 & 0 & 0 & 0 & 0 & 0 & 0 & 0 & 0 & 2 & 0 & 0 & 0 & 0 \\
\hline & Lvf & Lítico volcánico felsítico & 19 & 9 & 17 & 19 & 12 & 8 & 20 & 12 & 20 & 13 & 15 & 17 & 23 & 10 & 20 \\
\hline & D & Minerales densos & 0 & 2 & 6 & 4 & 4 & 2 & 3 & 1 & 3 & 4 & 0 & 1 & 6 & 3 & 2 \\
\hline & M & Matriz & 8 & 14 & 21 & 30 & 26 & 34 & 29 & 31 & 9 & 25 & 18 & 1 & 10 & 24 & 23 \\
\hline & $\mathbf{C}$ & Cemento calcítico & 19 & 13 & 11 & 0 & 6 & 0 & 7 & 0 & 11 & 2 & 5 & 5 & 0 & 4 & 0 \\
\hline & Mi & Micas & 11 & 7 & 5 & 0 & 4 & 0 & 10 & 9 & 2 & 8 & 9 & 0 & 16 & 11 & 0 \\
\hline & Total & & 559 & 555 & 596 & 507 & 552 & 494 & 571 & 552 & 575 & 575 & 556 & 499 & 579 & 578 & 502 \\
\hline
\end{tabular}


Tabla 3. Características principales de las tres petrofacies en $(*)$ medias aritméticas y desviaciones estándar. $(*)=$ parámetros recalculados al $100 \%$.

\begin{tabular}{|c|c|c|c|c|c|c|c|}
\hline Petrofacies & QFL* & QmFLt* & QmPK* & Rg-Rs-Rm* & $\mathbf{Q p} / \mathbf{Q m *}$ & $\mathrm{Qm}<5^{\circ} / \mathrm{Qm}>5^{\circ *}$ & $\mathbf{P} / \mathbf{F}^{*}$ \\
\hline & $\mathrm{Q}=59 \pm 6$ & $\mathrm{Qm}=54 \pm 5$ & $\mathrm{Qm}=64 \pm 6$ & $\mathrm{Rg}=24 \pm 18$ & & & \\
\hline 1.Potrero & $\mathrm{F}=28 \pm 6$ & $\mathrm{~F}=30 \pm 6$ & $\mathrm{P}=20 \pm 5$ & $\mathrm{Rs}=75 \pm 18$ & $0.52 \pm 0.085$ & $3.8 \pm 1.4$ & $1.35 \pm 0.56$ \\
\hline \multirow[t]{2}{*}{ Prieto } & $\mathrm{L}=13 \pm 7$ & $\mathrm{Lt}=16 \pm 6$ & $K=16 \pm 4$ & $\mathrm{Rm}=1 \pm 2$ & & & \\
\hline & $\mathrm{Q}=63 \pm 6$ & $\mathrm{Qm}=57 \pm 6$ & $\mathrm{Qm}=69 \pm 6$ & $\mathrm{Rg}=40 \pm 11$ & & & \\
\hline \multirow[t]{3}{*}{ 2.Rayones } & $\mathrm{F}=24 \pm 4$ & $\mathrm{~F}=26 \pm 4$ & $\mathrm{P}=20 \pm 4$ & $\mathrm{Rs}=55 \pm 12$ & $0.22 \pm 0.09$ & $3 \pm 10.68$ & $1.99 \pm 1.38$ \\
\hline & $\mathrm{L}=14 \pm 3$ & $\mathrm{Lt}=17 \pm 4$ & $K=11 \pm 4$ & $\mathrm{Rm}=5 \pm 9$ & & & \\
\hline & $\mathrm{Q}=61 \pm 4$ & $\mathrm{Qm}=56 \pm 3$ & $\mathrm{Qm}=64 \pm 4$ & $\mathrm{Rg}=40 \pm 7$ & & & \\
\hline \multirow[t]{2}{*}{ 3.Galeana } & $\mathrm{F}=29 \pm 4$ & $\mathrm{~F}=31 \pm 3$ & $\mathrm{P}=21 \pm 3$ & $\mathrm{Rs}=56 \pm 8$ & $0.22 \pm 0.05$ & $2.9 \pm 0.9$ & $1.33 \pm 0.37$ \\
\hline & $\mathrm{L}=10 \pm 2$ & $\mathrm{Lt}=13 \pm 2$ & $\mathrm{~K}=15 \pm 2$ & $\mathrm{Rm}=4 \pm 3$ & & & \\
\hline
\end{tabular}

\begin{tabular}{|c|c|c|c|c|c|c|c|c|c|c|c|c|c|}
\hline Muestra & & $\begin{array}{l}\text { Gn- } \\
1\end{array}$ & $\begin{array}{c}\text { Gn- } \\
4\end{array}$ & $\begin{array}{c}\text { Gn- } \\
6\end{array}$ & $\begin{array}{c}\text { Gn- } \\
9\end{array}$ & $\begin{array}{r}\text { Gn- } \\
10\end{array}$ & $\begin{array}{c}\text { Gn- } \\
11\end{array}$ & $\begin{array}{c}\text { Gn- } \\
21\end{array}$ & $\begin{array}{c}\text { Gn- } \\
24\end{array}$ & $\begin{array}{r}\text { Gn } \\
27\end{array}$ & $\begin{array}{c}\text { Gn- } \\
33\end{array}$ & $\begin{array}{r}\text { Gn- } \\
39 \\
\end{array}$ & \\
\hline $\mathbf{Q t} \%$ & & 2.2 & 64.0 & 61.7 & 61.7 & 53.7 & 62.0 & 59.4 & 64.6 & 57.1 & 63.6 & 58.8 & \\
\hline F\% & & 5.7 & 27.9 & 26.8 & 27.1 & 37.9 & 29.9 & 26.6 & 26.0 & 29.8 & 25.7 & 33.0 & \\
\hline Lt $\%$ & & 2.1 & 8.1 & 11.6 & 11.2 & 8.4 & 8.1 & 14.0 & 9.4 & 13.1 & 10.6 & 8.2 & \\
\hline Qm\% & & 7.6 & 57.5 & 54.0 & 54.2 & 51.6 & 56.9 & 53.5 & 59.2 & 52.7 & 58.8 & 54.2 & \\
\hline F\% & & 7.7 & 31.1 & 30.1 & 30.5 & 39.6 & 32.1 & 28.8 & 28.4 & 32.0 & 27.9 & 34.7 & \\
\hline $\mathbf{L t} \%$ & & 4.7 & 11.4 & 15.9 & 15.3 & 8.8 & 11.0 & 17.6 & 12.4 & 15.2 & 13.3 & 11.0 & \\
\hline Qm\% & & 7.5 & 64.9 & 64.2 & 63.9 & 56.6 & 63.9 & 65.0 & 67.6 & 62.2 & 67.8 & 60.9 & \\
\hline K\% & & 3.3 & 10.9 & 10.4 & 14.4 & 19.2 & 11.6 & 14.9 & 13.4 & 15.2 & 15.4 & 16.5 & \\
\hline P\% & & 9.2 & 24.2 & 25.4 & 21.7 & 24.2 & 24.5 & 20.2 & 19.0 & 22.6 & 16.8 & 22.6 & \\
\hline Rg\% & & 7.5 & 78.9 & 89.7 & 46.4 & 100. & 37.5 & 96.4 & 88.2 & 77.5 & 66.7 & 88.5 & \\
\hline Rs\% & & 2.5 & 0.0 & 6.9 & 48.2 & 0.0 & 60.4 & 0.0 & 0.0 & 0.0 & 25.0 & 7.7 & \\
\hline $\mathbf{R m} \%$ & & .0 & 21.1 & 3.4 & 5.4 & 0.0 & 2.1 & 3.6 & 11.8 & 22.5 & 8.3 & 3.8 & \\
\hline $\mathbf{P} / \mathbf{F}$ & & .33 & 2.22 & 2.37 & 1.47 & 1.26 & 2.00 & 1.31 & 1.36 & 1.42 & 1.06 & 1.30 & \\
\hline Muestra & $\begin{array}{c}\text { PP- } \\
02\end{array}$ & $\begin{array}{c}\mathrm{PP} \\
04\end{array}$ & $\begin{array}{l}\text { P- } \\
4\end{array}$ & $\begin{array}{c}\text { PP- } \\
05\end{array}$ & PP-6 & $\begin{array}{c}\text { PP- } \\
7\end{array}$ & PP-8 & $\begin{array}{c}\text { PP- } \\
11\end{array}$ & $\begin{array}{c}\text { PP- } \\
12\end{array}$ & $\begin{array}{c}\text { PP- } \\
13\end{array}$ & $\begin{array}{c}\text { PP- } \\
14\end{array}$ & $\begin{array}{c}\text { PP- } \\
15\end{array}$ & $\begin{array}{c}\text { PP- } \\
16\end{array}$ \\
\hline $\mathbf{Q t} \%$ & 44.6 & 53.6 & & 51.7 & 64.3 & 58.0 & 66.8 & 55.1 & 60.4 & 61.8 & 60.7 & 55.5 & 66.7 \\
\hline F\% & 18.8 & 33.4 & & 39.8 & 26.7 & 35.0 & 17.1 & 32.1 & 26.7 & 27.7 & 23.3 & 31.5 & 26.6 \\
\hline $\mathbf{L t} \%$ & 36.6 & 13.0 & & 8.5 & 9.0 & 7.1 & 16.0 & 12.8 & 12.9 & 10.5 & 16.0 & 13.0 & 6.7 \\
\hline$\underline{Q m \%}$ & 44.2 & 49.3 & & 48.7 & 58.3 & 53.5 & 63.7 & 48.8 & 56.2 & 56.2 & 52.9 & 50.5 & 63.0 \\
\hline$F \%$ & 18.9 & 35.4 & & 41.5 & 29.8 & 37.3 & 17.9 & 34.0 & 29.0 & 30.1 & 26.5 & 34.9 & 27.7 \\
\hline Lt $\%$ & 36.9 & 15.3 & & 9.8 & 11.9 & 9.2 & 18.4 & 17.2 & 14.8 & 13.7 & 20.6 & 14.6 & 9.3 \\
\hline Qm\% & 70.0 & 58.2 & & 54.0 & 66.2 & 58.9 & 78.1 & 58.9 & 66.0 & 65.1 & 66.6 & 59.1 & 69.5 \\
\hline K\% & 12.0 & 18.6 & & 13.6 & 11.8 & 21.5 & 9.6 & 18.3 & 15.1 & 17.8 & 8.6 & 18.7 & 12.9 \\
\hline $\mathbf{P} \%$ & 18.0 & 23.2 & & 32.4 & 22.1 & 19.6 & 12.3 & 22.8 & 18.9 & 17.1 & 24.9 & 22.2 & 17.7 \\
\hline Rg\% & 0.0 & 8.6 & & 15.2 & 0.0 & 22.2 & 0.0 & 31.8 & 23.1 & 30.2 & 46.5 & 34.7 & 0.0 \\
\hline Rs\% $\%$ & 99.3 & 91.4 & & 84.8 & 100.0 & 77.8 & 100.0 & 65.2 & 76.9 & 69.8 & 46.5 & 65.3 & 100.0 \\
\hline $\mathbf{R m} \%$ & 0.7 & 0.0 & & 0.0 & 0.0 & 0.0 & 0.0 & 3.0 & 0.0 & 0.0 & 7.0 & 0.0 & 0.0 \\
\hline $\mathbf{P} / \mathbf{F}$ & 1.50 & 1.25 & & 2.38 & 1.88 & 0.91 & 1.29 & 1.24 & 1.25 & 0.86 & 2.84 & 1.19 & 1.38 \\
\hline Muestra & & R-16 & & R-18 & $\mathrm{R}-1$ & & R-20 & R-21 & & -22 & R-23 & R-24 & \\
\hline $\mathrm{Qt} \%$ & & 64.9 & & 58.6 & 60.0 & & 69.1 & 74.4 & 63.5 & & 61.8 & 62.5 & \\
\hline F\% & & 23.4 & & 26.4 & 32.1 & & 18.4 & 14.5 & 23.5 & & 23.9 & 22.7 & \\
\hline $\mathbf{L t} \%$ & & 11.8 & & 15.0 & 7.9 & & 12.5 & 11.0 & 13.0 & & 14.3 & 14.8 & \\
\hline Qm\% & & 60.1 & & 51.1 & 56.6 & & 62.6 & 69.8 & 57.3 & & 54.6 & 55.0 & \\
\hline F\% & & 24.0 & & 29.0 & 33.1 & & 20.6 & 15.8 & 25.9 & & 26.3 & 24.8 & \\
\hline Lt $\%$ & & 15.9 & & 19.9 & 10.3 & & 16.8 & 14.4 & 16.8 & & 19.1 & 20.1 & \\
\hline Qm\% & & 71.5 & & 63.8 & 63.1 & & 75.2 & 81.5 & 68.8 & & 67.5 & 68.9 & \\
\hline K\% & & 3.5 & & 12.4 & 12.7 & & 9.9 & 8.1 & 10.5 & & 12.1 & 10.6 & \\
\hline P\% & & 25.0 & & 23.8 & 24.2 & & 14.9 & 10.4 & 20.7 & & 20.5 & 20.5 & \\
\hline Rg\% & & 51.6 & & 59.6 & 44.1 & & 28.6 & 33.3 & 25.5 & & 50.5 & 46.7 & \\
\hline Rs\% & & 37.4 & & 35.1 & 54.4 & & 68.3 & 66.7 & 70.6 & & 46.2 & 53.3 & \\
\hline $\mathbf{R m} \%$ & & 11.0 & & 5.3 & 1.5 & & 3.2 & 0.0 & 3.9 & & 3.2 & 0.0 & \\
\hline $\mathbf{P} / \mathbf{F}$ & & 6.9 & & 1.8 & 1.8 & & 1.5 & 1.3 & 2.0 & & 1.5 & 1.6 & \\
\hline
\end{tabular}

moscovita, hematita y circones en cantidades menores que las observadas en la petrofacies A. El contenido de matriz es menor al $10 \%(\sim 4.5 \%)$, mientras que el cemento es calcita que muestra un hábito poikilotípico $(>10 \%)$.

\subsection{Petrofacies C: Área de Galeana "Meta- plutonoclástica"}

Dentro del esquema de McBride (1963), las areniscas de la petrofacies $\mathrm{C}$ son clasificadas como arcosas que van de moderadamente a bien clasificadas $\left(\mathrm{Q}_{61} \mathrm{~F}_{29} \mathrm{~L}_{10}\right.$; McBride, 1963; Figura 5). El constituyente más abundante es el cuarzo monocristalino que se presenta en granos moderadamente redondeados, exhibe una mayor proporción de extinción recta que ondulante. Los cuarzos monocristalinos muestran bordes de crecimiento sintaxial (Figura 6F). El cuarzo policristalino constituido por más de tres cristales exhibe texturas tectónicas caracterizadas por mostrar contactos suturados y extinción ondulante grano a grano, $\mathrm{y}$ algunas veces con moscovita asociada (Figura 7A, 7B y 7C). Los feldespatos son de tipo: (1) feldespatos alcalinos, subangulosos a subredondeados con maclado tipo carlsbad, parcialmente remplazados por caolinitasericita, siendo poco frecuentes las microclinas; y (2) plagioclasas sódicas, subangulosas a subredondeadas con maclado tipo polisintético y combinado, predominando la albita (Figura 7D y 7E). En esta petrofacies el contenido de feldespato potásico es mayor que en las otras petrofacies $(\mathrm{P} / \mathrm{K}=1.33 \pm 0.37$; Tablas 3 y 4$)$. Los fragmentos líticos son subredondeados a redondeados, conformados por areniscas 




Figura 5. Esquema ternario QFL para clasificar areniscas de McBride (1963). de grano fino, lutitas, fragmentos volcánicos con texturas lathworth, felsíticas y raros microlíticos (Figura 7D y 7F), líticos metamórficos con texturas metapsamíticas/felsíticas ${ }_{2}$ y metapsamíticas/felsíticas ${ }_{3}$, y fragmentos de rocas con texturas faneríticas de composiciones granodioríticas y graníticas (Figura 7E).

Los minerales accesorios son moscovita y biotita. Los minerales pesados muestran forma romboédrica, posiblemente hematita y circones. La matriz se encuentra constituida por arcillas, variando en su contenido de 0.2 al $6.9 \%$.

\subsection{Composición del área fuente}

La composición del o las áreas fuentes se evaluó empleado metodologías de diversos autores (e.g. Basu et al., 1975; Arribas et al., 1990; Marsaglia, 1991; Johnson, 1993), quienes documentan la composición de áreas fuentes de acuerdo a criterios como: (i) variaciones en los tipos de cuarzos; (ii) concentración relativa de diferentes fragmentos líticos; y (iii) las relaciones que presentan las plagioclasas versus el feldespato-K.

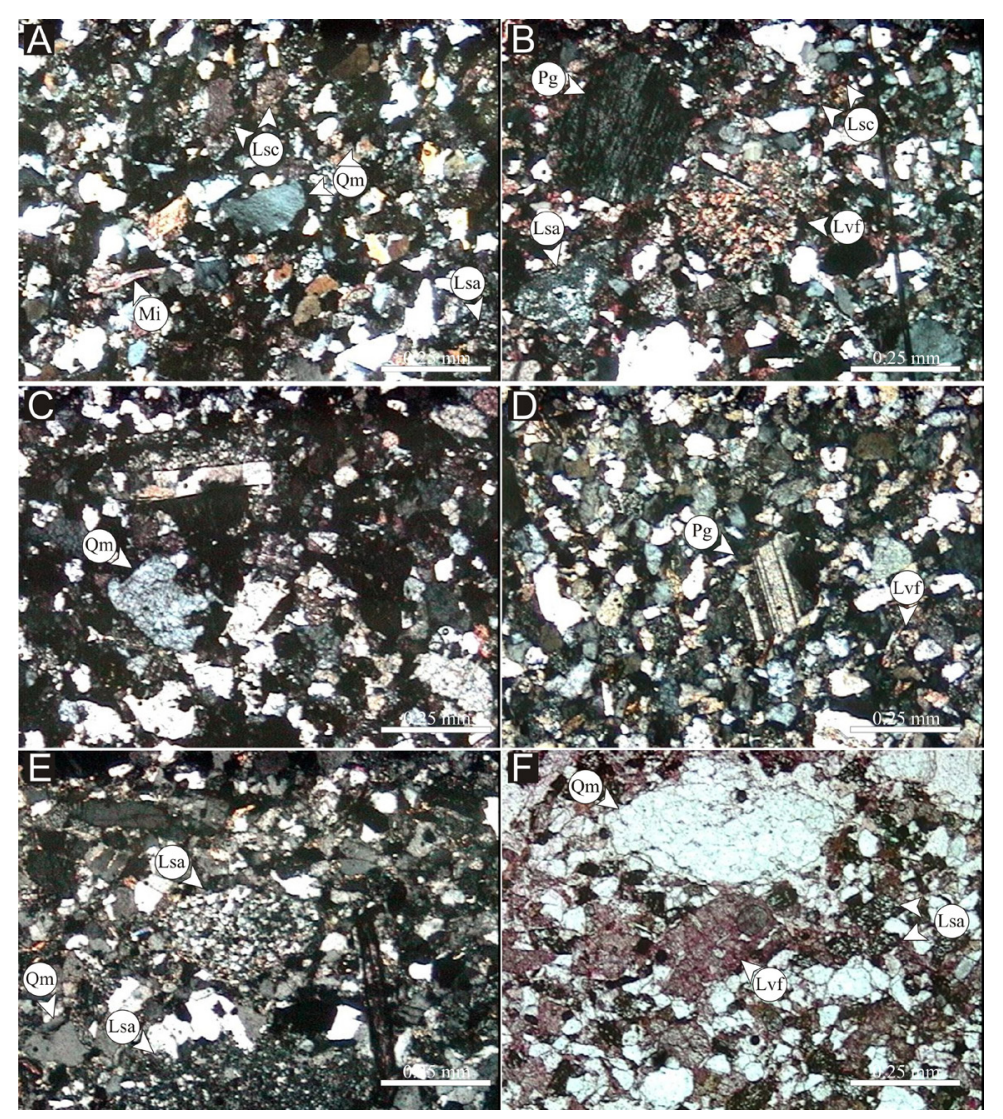

Figura 6. Fotomicrografías de las petrofacies A, B y C. Nícoles paralelos. (A). Arcosa de la petrofacies A (PP-8) mostrando: líticos sedimentarios carbonatados (Lsc), cuarzo monocristalino (Qm), micas (Mi) y líticos sedimentarios de areniscas; (B) Arcosa lítica de la petrofacies A (PP-21), muestra líticos volcánicos felsíticos (Lvf), líticos sedimentarios carbonatados (Lsc), líticos sedimentarios de areniscas (Lsa) y cristales del tamaño de las arenas medias de plagioclasa (Pg). (C) Arcosa lítica de la petrofacies B (R24), cuarzo monocristalino de origen volcánico (Qm); (D) Subarcosa lítica, petrofacies B, observe las plagioclasas del tamaño de las arenas medias (Pg) y el abundante cuarzo monocristalino (R23); (E) Arcosa lítica, petrofacies B (R9a), muestra el alto contenido de líticos sedimentarios de areniscas (Lsa); (F) Arcosa, petrofacies C (Gn40), muestra un cuarzo monocristalino con bordes de crecimiento syntaxiales de cuarzo. 


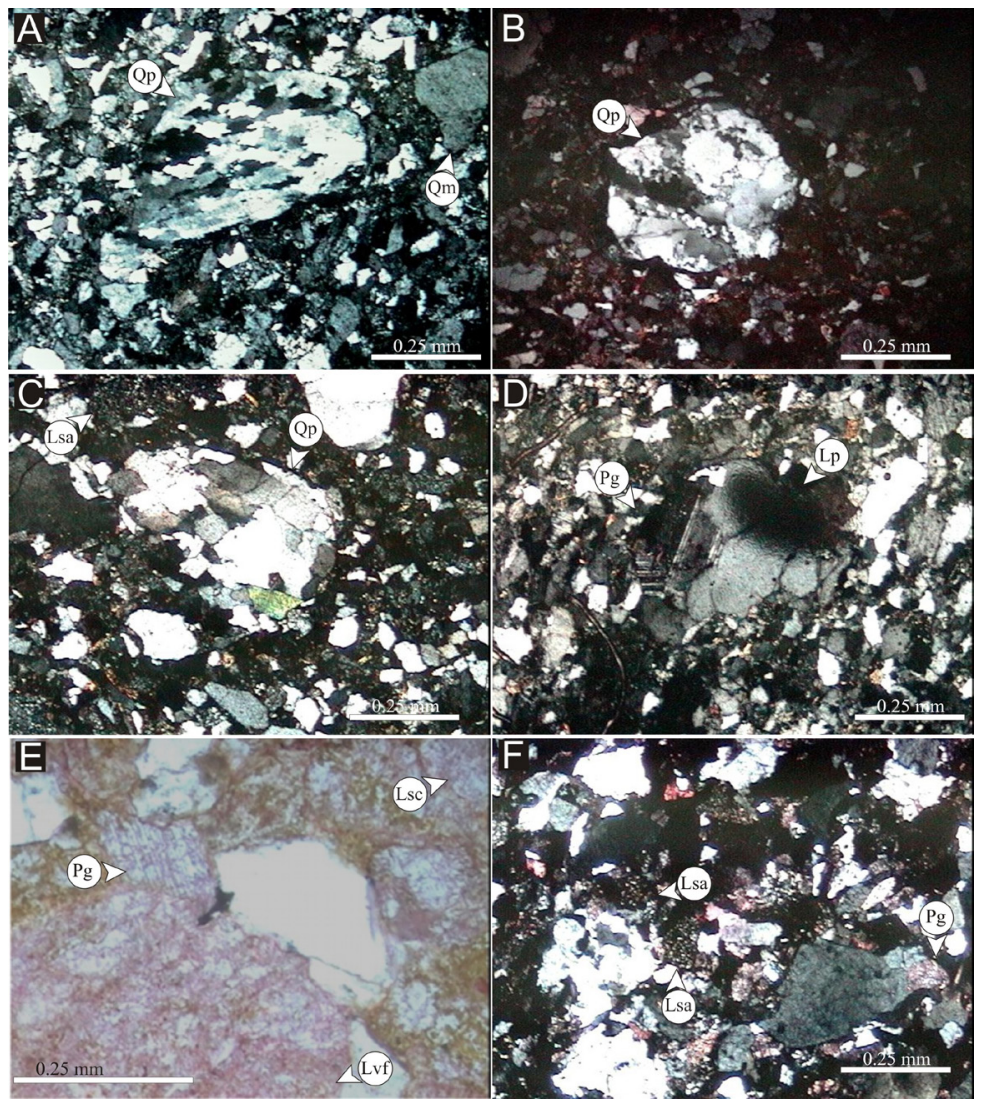

Figura 7. Fotomicrografías de la petrofacies C. (A) y (B) Arcosa que presenta cuarzo policristalino con textura tectónica (Gn9 y Gn 24, respectivamente); (C) Arcosa que exhibe cuarzo policristalino (Qp) con textura de alto grado y líticos sedimentarios de areniscas (Lsa; Gn81); (D) Lítico plutónico, posiblemente de composición granítica o granodiorítica, obsérvese la existencia de las plagioclasas (Pg), con el cuarzo monocristalino (Qm; Gn39); (E) Arcosa en nícoles paralelos, en la que se presentan líticos volcánicos felsíticos (Lvf), plagioclasas (Pg), y líticos sedimentarios carbonatados (Lsc); (F) Arcosa lítica, en la que abundan los líticos sedimentarios de areniscas (Lsa; Gn51).

\subsubsection{Cuarzos}

Basu et al. (1975) emplea la relación que presentan los diferentes tipos de cuarzo (e.g., policristalinos con monocristalinos) y el tipo de extinción que exhiben (recta u ondulante), para documentar la composición del área fuente. Estos autores proponen un diagrama con forma de diamante que muestra estas características (Qmo, Qmr, Qp $<3$, y $\mathrm{Qp}>3$; Tablas 1-3; Figura 8). En este diagrama, las muestras analizadas sugieren procedencias de rocas graníticas y rocas metamórficas de grado medio a alto (Figura 8).

Los tipos de cuarzos registran como áreas fuente: (1) cuarzo con extinción no-ondulante y estrías, el cual en las muestras analizadas está posiblemente relacionado con áreas fuente plutónicas; (2) cuarzo metamórfico (policristalino con fábrica tectónica), que sugiere una fuente de rocas metamórficas de grado bajo a medio (Figura 7A); (3) cuarzo volcánico con golfos de corrosión, registra procedencias de rocas volcánicas de composición intermedia; (4) cuarzo policristalino con biotita asociada, sugiere procedencias de rocas metamórficas de alto grado (Figura 7C); (5) y cuarzos con bordes de crecimiento sintaxial que pueden representar cuarzos de segundo ciclo de sedimentación relacionados con los procesos de reciclamiento, como ha sido documentado por Blatt (1967) y Mokowitz y Sibley (2001).

En el diagrama ternario que compara los parámetros Qmr-Qmo-Qp propuesto por Arribas et al. (1990; Figura 9 y Tablas 2 y 3 ), las muestras de las tres petrofacies se ubican sobre el vértice en la parte central del triángulo. Sin embargo, las muestras de la petrofacies A presentan un mayor enriquecimiento de cuarzo policristalino.

\subsubsection{Feldespatos}

Las plagioclasas predominan en todas las petrofacies, con relaciones que van de 0.5-2.8 (P/K), evidenciando rocas fuentes plutónicas de composiciones variables. Las muestras con relaciones de $\mathrm{P} / \mathrm{K}>1$ registran fuentes de composiciones plutónicas variables (e.g., granitoides), mientras que muestras con valores de $\mathrm{P} / \mathrm{K}<1$ indican procedencias de rocas de composición granodiorítica como lo han documentado Marsaglia (1991) y Critelli y Nilsen (2000) (Tabla 3; Figura 10).

La presencia de microclina sugiere una fuente de composición granítica o granodiorítica. En el caso del miembro arenoso Galeana, el bajo contenido de feldespatos potásicos se interpreta como resultado de desintegración por procesos de transporte (cf. Pittman, 1969; Critelli et al., 


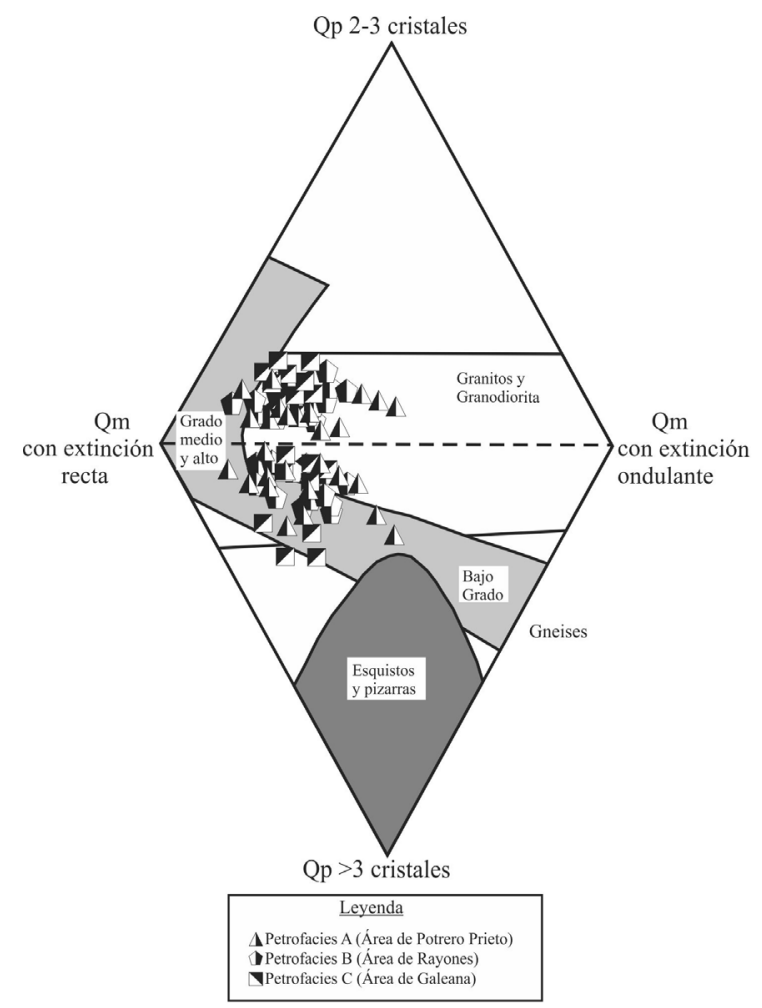

Figura 8. Diagrama en forma de diamante que enfrenta a los diferentes tipos de cuarzos "Qp-Qm no ondulante-Qm ondulante-Qp" de Tortosa et al., (1991).

2002) o por la alteración a minerales arcillosos debido a procesos de diagénesis tardía (caolinita o sericita).

\subsubsection{Fragmentos Líticos}

Los fragmentos líticos son los constituyentes más inestables de las rocas clásticas, por consiguiente presentan una menor resistencia al grado de transporte y tipos de intemperismo al que fueron expuestos (Blatt, 1967; Cameron y Blatt, 1971; Suttner et al., 1981; Johnson, 1993; Nesbitt et al., 1997). Las tres petrofacies muestran un contenido modal en fragmentos líticos muy similar (Tabla 3). Sin embargo, presentan variaciones significativas en los diferentes fragmentos líticos (Tabla 2).

Los líticos volcánicos sugieren como área fuente rocas volcánicas de composición andesítica-riolítica. Los líticos volcánicos con texturas lathwork, felsíticas y microlíticas evidencian rocas volcánicas de composiciones andesíticasrioliticas (Dickinson, 1970), siendo más concentrados en la petrofacies $\mathrm{C}$.

Los fragmentos líticos sedimentarios están constituidos por fragmentos de areniscas de grano fino, fragmentos de lutitas, así como fragmentos de calizas con texturas micríticas y esparíticas, reflejando el reciclamiento de la cubierta sedimentaria (e.g., formaciones Minas Viejas, Zuloaga, La Casita y Taraises) y la erosión contemporánea asociada a procesos de alta energía (e.g., oleaje, mareas) al momento del depósito.

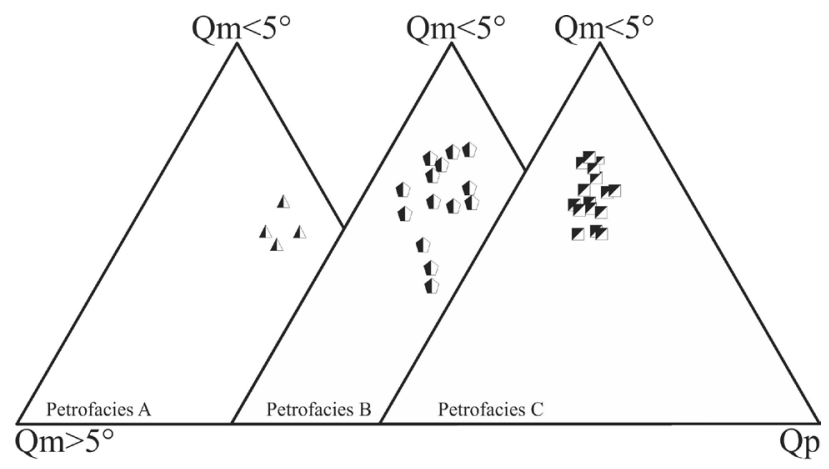

Figura 9. Diagrama ternario que compara los cuarzos del tipo $\mathrm{Qm}<5^{\circ} \mathrm{Qm}$ $>5^{\circ} \mathrm{Qp}$ (tomado de Arribas et al., 1990).

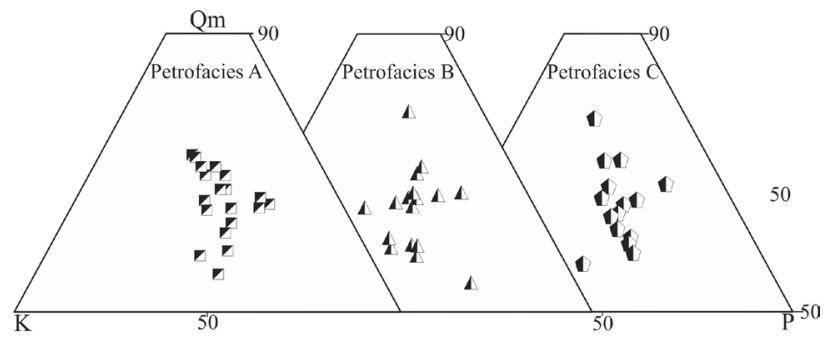

Figura 10. Diagrama triangular que compara los cuarzos monocristalinos con las plagioclasas y feldespatos (tomada de Dickinson y Suczek, 1979). Nótese que todas la petrofacies se muestran más enriquecidas en plagioclasas que en feldespatos alkalinos.

Los fragmentos líticos metamórficos con texturas metapsamíticas/felsíticas ${ }_{2}$ y metapsamíticas/felsíticas ${ }_{3}$ provienen de rocas metamórficas de grado bajo a medio como son esquistos de moscovita.

Los líticos plutónicos con texturas faneríticas corresponden a rocas cristalinas de composición granítica o granodiorítica.

El diagrama ternario $\mathrm{Rg}-\mathrm{Rs}-\mathrm{Rm}$ de Critelli et al. (2002) sugiere que la petrofacies A tiene mayor influencia de ensambles sedimentarios (e.g. lutitas, areniscas-Rs), mientras que las petrofacies $\mathrm{B}$ y $\mathrm{C}$ muestran una composición mixta del ensamble granoso con el ensamble sedimentario con poca adición de los ensambles metamórficos (e.g. granitos, gneises-Rg; Figura 11).

\subsection{Modos detríticos y procedencia de las areniscas}

Dickinson y Suczek (1979) y Dickinson et al., (1983), por medio de un análisis petrográfico basado en la composición de areniscas de diferentes ambientes tectónicos, proponen dos diagramas ternarios que permiten tener una aproximación al ambiente tectónico en que fueron depositadas (diagramas QFL y QmFLt; Tablas 2-4). Dentro del diagrama QmFLt, la petrofacies A se ubica en los campos de "transición continental-mezcla", la petrofacies B en los límites mezcla-cuarzoso reciclado y la petrofacies $\mathrm{C}$ dentro del campo de transición continental (Figura 12). 


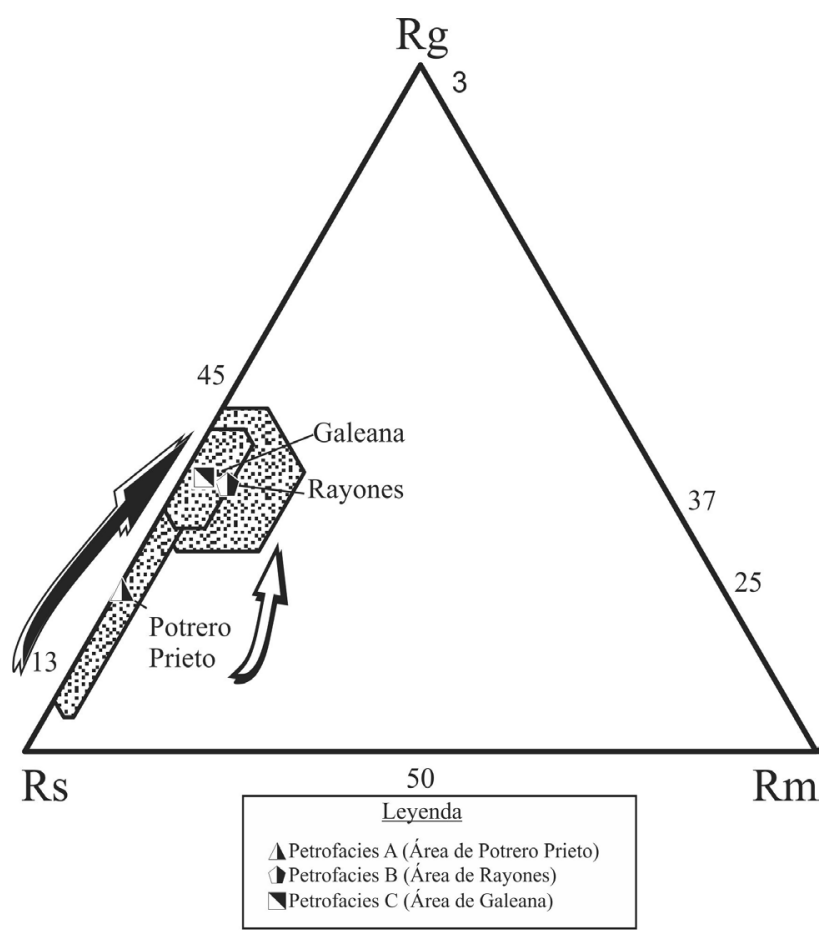

Figura 11. Diagrama ternario que compara los ensambles granosossedimentarios y metamórficos de Critelli y Le Pera (1995), elaborado para cuantificar la exhumación de rocas de la corteza media. Los polígonos representan la desviación estándar respecto de la media aritmética. Las flechas marcan las posibles tendencias de enriquecimiento de la parte más proximal a la distal.

Garzanti et al. (2001) y Marsaglia et al. (2007) proponen un diagrama QFL actualista en ambientes de tipo rift. Dentro de este diagrama las muestras de las petrofacies A y $\mathrm{C}$ se ubican dentro del campo de rift disectado (Figura 13), mientras que las muestras de la petrofacies B se grafican en el límite del campo de rift disectado con tendencias al rift no disectado con influencia de orógeno o sutura.

\section{Discusión y Conclusiones}

La composición modal de las areniscas del miembro arenoso Galeana muestra la evidencia de dos ciclos de "procedencia principales" o secuencias de procedencia, de acuerdo con las características petrográficas observadas. Estos ciclos pudieran asociarse a eventos tectónicos regionales contemporáneos al depósito del miembro arenoso Galeana. Las tres petrofacies documentadas dentro de las areniscas del miembro arenoso Galeana permiten proponer la evolución petrotectónica de la porción sur de la Fosa de Monterrey durante el Cretácico Temprano. Ocampo-Díaz (2007) divide al miembro arenoso Galeana en tres secuencias deposicionales con características sedimentológicas distintas:

1. La petrofacies A "primera secuencia de procedencia" está constituida por arcosas líticas, moderadamente

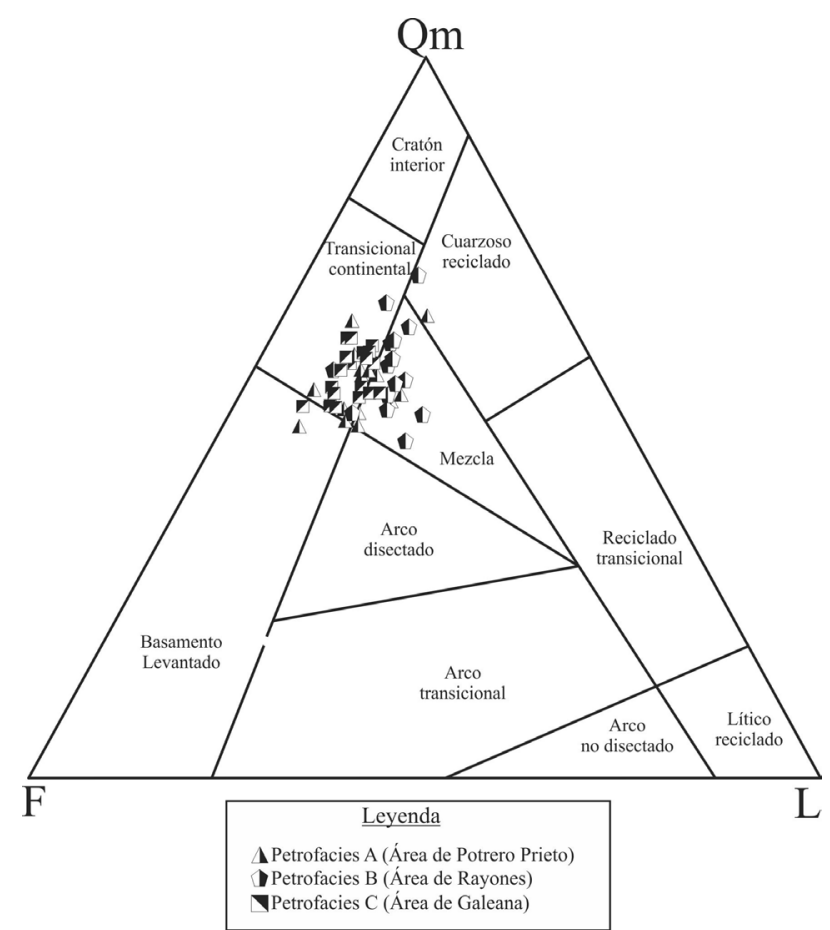

Figura 12. Diagrama ternario Qm-F-L para discriminar ambientes tectónicos de Dickinson et al (1983).

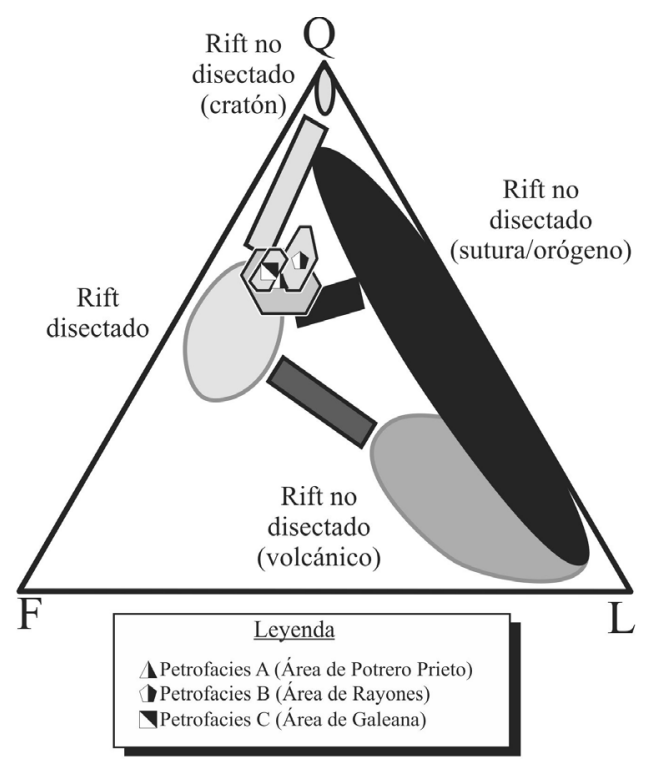

Figura 13. Diagrama triangular Q-F-L de Garzanti et al. (2001), modificado por Marsaglia et al., (2007), para determinar el ambiente tectónico en secuencias relacionadas a rompimiento.

clasificadas $\left(\mathrm{Q}_{59} \mathrm{~F}_{28} \mathrm{~L}_{13}\right)$, desarrolladas durante el depósito de las facies mareales. Esta petrofacies representa la erosión de la cubierta sedimentaria conformada por rocas marinas del pre-Cretácico Temprano (e.g., formaciones Minas Viejas, Zuloaga, La Casita), como lo indica el alto contenido de fragmentos líticos carbonatados y lutitas. La relación alta de Qp/Qm sugiere áreas fuentes metamórficas de grado bajo, 
mostrando poca influencia de rocas fuentes volcánicas y/o plutónicas. El incremento de cuarzo monocristalino con o sin bordes de sobre-crecimientos sintaxiales hacia la cima de la secuencia, evidencía que la composición modal de la petrofacies A pudo ser afectada por procesos de selección y madurez relacionados con procesos mareales, como lo ha documentado Spencer (1963), Balazs y de Vries (1972), Parrado Román y Achab (1999) en sedimentos mareales de la Península Ibérica, y Ocampo-Díaz et al. (2008) dentro del miembro arenoso Galeana.

2. Las areniscas de las Petrofacies B son subarcosas líticas $\left(\mathrm{Q}_{63} \mathrm{~F}_{24} \mathrm{~L}_{14}\right.$; “primera secuencia de procedencia") que corresponden a los depósitos de dunas subacuosas (Ocampo-Díaz, 2007). La composición de las áreas fuente de esta petrofacies es más variada que la de la petrofacies A. Se pueden postular las siguientes fuentes: (1) rocas de composición andesítica-riolítica, como lo indica la alta relación de $\mathrm{P} / \mathrm{K}$ y los líticos volcánicos con texturas felsítica, lathworth y microlíticos con texturas fluidales; (2) rocas sedimentarias preexistentes de carbonatos, lutitas y principalmente areniscas que pueden ser interpretadas como la erosión de rocas sedimentarias jurásicas marinas, mientras que los líticos de areniscas pueden representar el reciclamiento interno dentro de los depósitos de dunas subacuosas; (3) las variaciones entre el Qp/Qm y Qm < 5\% $\mathrm{Qm}>5^{\circ}$, junto con el comportamiento de enriquecimiento y empobrecimiento vertical en el contenido de Qm, evidencian un reciclamiento interno mayor ( $c f$. Visher, 1969; Balazs y de Vries, 1972; Anfuso et al., 1999; Ocampo-Díaz, 2007). Entre tanto, los cuarzos indican fuentes graníticas, granodioríticas y rocas metamórficas de grado bajo a medio.

3. La petrofacies $C$ está formada por arcosas $\left(\mathrm{Q}_{61} \mathrm{~F}_{29} \mathrm{~L}_{10}\right)$ correspondientes con los depósitos del frente deltaico y sublitorales del área de Galeana de la "segunda secuencia de procedencia". La composición de las áreas fuente postuladas para esta petrofacies son: (1) graníticas o granodioríticas, registradas por los fragmentos líticos con texturas faneríticas y por feldespatos de variedad microclina con maclado en enrejado; (2) fuentes metamórficas de grado bajo a medio como lo indica la presencia de cuarzos policristalinos con más de 3 cristales con texturas tectónicas y líticos metapsamíticos/felsíticos ${ }_{2}$ y metapsamíticos/felsíticos 3 ; (3) los fragmentos líticos sedimentarios de areniscas, lutitas y calizas, que indican erosión de rocas sedimentarias preexistentes, así como también el reciclamiento alogenético de las petrofacies A y B, como sugieren los cuarzos monocristalinos con bordes de crecimiento sintaxiales que marcan sedimentos de segundo ciclo de sedimentación; y (4) rocas de composición andesítica-riolítica, como lo demuestra la presencia de fragmentos de líticos volcánicos felsíticos.

Las diferentes áreas fuentes inferidas sugieren que las areniscas del miembro arenoso Galeana proceden de bloques de basamento (diagramas QmFLt) que fueron expuestos durante el Valanginiano tardío-Hauteriviano medio. Este levantamiento se originó por fallas de basamento de componente normal, asociadas a grandes sistemas laterales de tipo strike-slip y pull-apart denominados como el Sistema McCoy-Sabinas dentro de un régimen transtensivo, favorecido por una subducción oblicua alojada en la margen paleo-pacífica (Figura 14A). Ocampo-Díaz (2011) sugiere que el régimen transtensivo favoreció el desarrollo de cuencas de tipo strike-slip y la exhumación de los bloques que bordeaban a la Fosa de Monterrey durante el Jurásico Tardío y Cretácico Temprano (e.g., Bloque de Coahuila, Archipiélago de Tamaulipas e Isla de Terán). En la región norte de la Fosa de Monterrey (sur del Bloque de Coahuila), la exhumación inicia durante el Kimmerigdiano tardío-Berriasiano y termina durante el Hauteriviano temprano, desarrollándose en tres fases. En tanto que en la
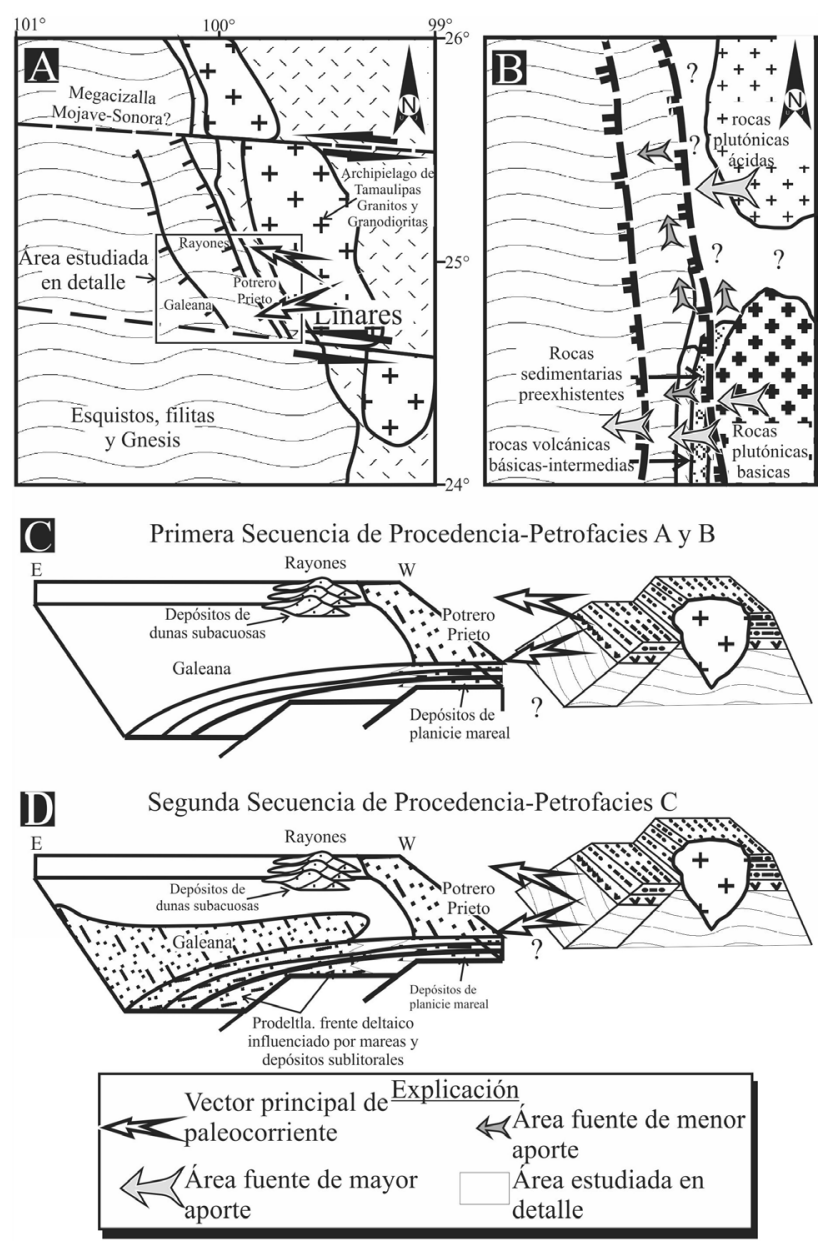

Figura 14. (A) Mapa regional de la distribución de las rocas del basamento (Tomado de Eguiluz de Antuñano, 2001); (B) Configuración de las rocas fuentes que dieron origen al miembro arenoso Galeana, marcada en el recuadro de la figura $\mathrm{A}$, nótese el cambio de área fuente (Modificada de Ocampo-Díaz, 2007); (C) Bloque diagramático de la posible ubicación del paleoelemento de la Isla de Terán, distribución de las facies durante el origen de la secuencia de procedencia 1 o petrofacies A y B (Las flechas indican la dirección de paleocorrientes medias, tomadas de Ocampo-Díaz, 2007); (D) Máxima exhumación del Archipiélago de Tamaulipas e Isla de Terán. Generación de la Secuencia de procedencia 2 o de la petrofacies C. Progradación de los depósitos mareales y dunas subacuosas a los sistemas deltaicos del área de Galeana 
región sur, los procesos de exhumación inician durante el Valanginiano temprano-medio, y se realizaron en dos fases de exhumación. La primera fase de levantamiento ocurrió durante el Valanginiano temprano-medio, generando la primera secuencia de procedencia (petrofacies A y B). Durante este lapso se erosionan principalmente las rocas sedimentarias preexistentes, volcánicas y escasamente plutónicas y metamórficas, (Ocampo-Díaz, 2007; OcampoDíaz, 2011), que conformaban la cubierta sedimentaria hasta ese momento (formaciones La Casita, Zuloaga, La Joya y Huizachal). Las diferencias presentes en la relación $\mathrm{P} / \mathrm{K}, \mathrm{Qp} / \mathrm{Qm}$ y RgRsRm (Tabla 3) en ambas petrofacies, indican áreas fuentes de composiciones distintas, como lo propone sedimentológica y geoquímicamente Ocampo-Díaz (2007) con los datos de paleocorrientes, el incremento en la madurez textural y las diferencias en las concentraciones geoquímicas de elementos estables (e.g. Ti/Nb y Zr/Sc). La segunda etapa de exhumación implica la exhumación de los bloques del basamento de composición granítica, granodiorítica y metamórfica de grado bajo a medio, depositándose la segunda secuencia de procedencia (petrofacies $\mathrm{C}$ ). La petrofacies $\mathrm{C}$ registra procedencias más complejas, conformadas esencialmente por rocas metamórficas de grado bajo a medio (Rm 4), graníticas y/o granodioríticas y volcánicas de composición intermedia ( $\operatorname{Rg} \sim 40$; Figura 11 y Tabla 3 ). Es posible que el incremento en el contenido de material volcánico, plutónico $(\mathrm{Rg})$ y metamórfico $(\mathrm{Rm})$, se encuentre relacionado con: 1) procesos de exhumación y erosión profunda de los bloques de basamento y 2) el incremento de la tasa de aporte de sedimentos que propició la progradación de depósitos de planicie mareal y dunas subacuosas (petrofacies A y B) a depósitos deltaicos y sublitorales (petrofacies C; Figuras 14B-D). Estos datos son concordantes con los resultados geoquímicos de Ocampo-Díaz (2007), que emplea relaciones geoquímicas sensitivas al grado de transporte y composición del área fuente (e.g. $\mathrm{Zr} / \mathrm{Sc}, \mathrm{SiO}_{2} / \mathrm{Al}_{2} \mathrm{O}_{3}, \mathrm{Na}_{2} \mathrm{O} /$ $\mathrm{K}_{2} \mathrm{O}, \mathrm{La} / \mathrm{Sc}$ y K/B), mostrando que: 1) las muestras del área de Rayones tienen el más alto grado de transporte, en tanto que las de Galeana y Potrero Prieto muestran grados intermedios y bajos, respectivamente; 2) las diferencias en la composición del área fuente en los casos analizados; y 3) los procesos de selección y clasificación dentro de los ambientes sedimentarios documentados no modificaron la naturaleza del área fuente. Casos similares sobre la evolución petrotectónica de sistemas de rift intraplaca, pullapart y strike-slip han sido documentados en la Cuenca de Cameros, en España por González-Acebrón et al. (2007) y Arribas et al. (2003); en el Mar Rojo, del sistema Aden, por Garzanti et al. (2001) y Garzanti et al. (2003); y en el Triásico del Nesocratón del Deseado, Argentina, por Jenchen (2001).

Durante la exhumación del Archipiélago de Tamaulipas e Isla de Terán (Michalzik, 1988; Ocampo-Díaz, 2007; Ocampo-Díaz, 2011) tuvieron lugar dos fases de sedimentación que implican: 1) erosión de la cubierta sedimentaria y escasas rocas del basamento y depósito de la secuencia de procedencia 1 ; y 2) la exposición completa de las rocas del basamento del Noreste de México dentro de la Fosa de Monterrey y el depósito de la secuencia de procedencia 2. Las diferencias observadas en las petrofacies indican variaciones composicionales en el área fuente durante la sedimentación.

Las características petrográficas y texturales de los fragmentos líticos de las relaciones de Qp/Qm, $\mathrm{P} / \mathrm{K}$, cuantificados en las tres petrofacies, sugieren una procedencia de: 1) rocas sedimentarias marinas pre-Jurásico Tardío; 2) rocas volcánicas de composición andesíticariolítica, relacionadas con magmatismo del Arco Continental de Nazas, o bien de los diques y sills, documentados en la Formación Huizachal; 3) basamento cristalino, de posible composición granítica o granodiorítica, reportado en las Islas de Terán y de Linares, y 4) aporte tanto del esquito de Granjeno que aflora en Ciudad Victoria, Tamaulipas, como de los esquistos psamíticos de grado bajo a medio que afloran en Aramberrí, Nuevo León (Figura 14B-14D).

El ambiente tectónico de una cuenca de tipo strike-slip es comprobado por los resultados de rift disectado obtenido del diagrama Q-F-L propuesto por Garzanti et al. (2001). De acuerdo con Reading (1980), Sylvester (1988), Nilsen y Sylvester $(1995,1999)$, los sistemas strike-slip y de rift inician con la erosión de las rocas que cubren el basamento, y su contínua evolución da la pauta de la exhumación de las rocas que conforman su basamento. Bajo este criterio, el campo de rift disectado propuesto por Garzanti et al. (2001), involucra el tiempo de mayor exhumación y erosión profunda de los bloques que conforman los basamentos dentro de una cuenca de tipo rift y de tipo strike-slip. Considerando lo descrito previamente, se propone que durante el Valanginiano temprano-Hauteriviano medio en la Fosa de Monterrey se efectuó la exhumación de las rocas que conforman el basamento por procesos transtensivos relacionados con el sistema de cuencas McCoy-Sabinas, favorecidas por un régimen de subducción oblicua alojado en la margen paleo-pacífica (Ocampo-Díaz, 2007; OcampoDíaz et al., 2008; Ocampo-Díaz, 2011).

\section{Agradecimientos}

El presente trabajo forma parte de las tesis de maestría y doctorado de Ocampo-Díaz, Y. Z. E; por ello agradece al CONACyT por la beca doctoral. Al Dr. Oscar Talavera Mendoza responsable del Laboratorio de Geoquímica de la Unidad Académica de Ciencias de la Tierra, Universidad Autónoma de Guerrero (LG-UACT-UAG), por su apoyo durante la elaboración del conteo de puntos. Se agradece al Dr. José Arribas, del Departamento de Petrología y Geoquímica, Universidad Complutense de Madrid, España, por las sugerencias con respecto al análisis de datos y artículos relacionados con el tema expuesto en el presente artículo. Al Laboratorio de Petrología 
Sedimentaria de la UACT, de la UAGro, por facilitar la toma de microfotografías que ilustran el artículo. A Rafael Barboza por la invitación para participar en el volumen especial conmemorativo al 50 aniversario de la UASLP. A Elena Centeno García y al revisor anónimo por su revisión crítica y comentarios que han enriquecido enormemente el contenido del manuscrito. Finalmente a Igor Rubio Cisneros y Margarita Martínez Paco por enriquecer la versión temprana del presente trabajo.

\section{Referencias}

Anfuso, G., Andrés, J., Vindel, B., Martinez, J.A., 1999, Morphometric characteristics and internal structures of intertidal bars on the northwest Cadiz littoral (southwestern Iberian Peninsula): Boletín del Instituto Español de Oceanografía, 15, 203-211.

Arribas, J., Alonso, A., Mas, R., Tortosa, A., Rodas, M., Barrenechea, J.F., Alonso-Azcárate, J., Artigas, R., 2003, Sandstone petrography of continental depositional sequences of an intraplate rift basin: Western Cameros Basin (North Spain): Journal of Sedimentary Research, 73, 309-327.

Arribas, J., Gómez-Gras, D., Rosell, J., Tortosa, A., 1990, Estudio comparativo entre las areniscas Paleozoicas y Triásicas de la Isla de Menorca: Evidencias de proceso de reciclado: Revista de la Sociedad Geológica de España, 3, 105-116.

Balazs, R.J., de Vries K,G., 1972, Roundness-mineralogical relations of some intertidal sands: Journal of Sedimentary Petrology, 42, 425-433.

Barboza-Gudiño, J.R., Orozco-Esquivel, M.T., Gómez-Anguiano M., Zavala-Mosiváis, A., 2008, The Early Mesozoic volcanic arc of western North America in northeastern Mexico: Journal of South American Earth Sciences, 25, 49-63.

Barboza-Gudiño, J.R., Tristán-González, M., Torres-Hernández, J.R., 1999, Tectonic setting of pre-Oxfordian units from central and northeastern Mexico: A review, en Bartolini, C., Wilson, J.L., Lawton, T.F. (eds.), Mesozoic sedimentary and tectonic history of north-central Mexico: Boulder, Colorado, Geological Society of America, Special Paper 340, 197-210.

Barboza-Gudiño, J.R., Tristán-González, M., Torres-Hernández, J.R., 1998, The Late Triassic-Early Jurassic active continental margin of western North America in northeastern Mexico: Geofísica Internacional, 37, 4, 283-292.

Bartolini, C., Lang, H., Cantú-Chapa, A., Barboza-Gudiño, R., 2001, The Triassic Zacatecas Formation in Central Mexico: Paleotectonic, Paleogeogtraphic, and Paleobiogeographic Implications, en Bartolini, C., Buffler, R.T., Cantú-Chapa, A., (eds.), The Western Gulf of Mexico Basin: Tectonics, Sedimentary Basins, and Petroleum Systems: Tulsa, lahoma, American Association of Petroleum Geologists, Memoir 75, 295-315.

Bassett, K.N., Busby, C.J, 2005, Tectonic setting of the Glance Conglomerate along the Sawmill Canyon fault zone, southern Arizona: A sequence analysis of an intra-arc strike-slip basin, en Anderson, T.H., Nourse, J.A., McKee, J.W., Steiner, M.B. (eds.). The Mojave-Sonora megashear hypothesis: development, assessment, and alternatives: Boulder, Colorado, Geological Society of America, Special Paper 393, 377-400.

Basu, A., 1976, Petrology of Holocene fluvial sand derived from plutonic source rocks; implications to paleoclimatic interpretation: Journal of Sedimentary Petrology, 46, 694-709.

Basu, A., Young, S.W., Suttner, L.J., James, W.C., Mack, G.H., 1975, Reevaluation of the use of undulatory extinction and polycrystallinity in detrital quartz for provenance interpretation: Journal of Sedimentary Petrology, 45, 873-882.

Blatt, H., 1967, Original characteristics of clastic quartz grains: Journal of Sedimentary Petrology, 37, 401-424.

Blatt, H., Christie, J.M., 1963, Udulatory extinction in quartz of igneous and metamorphic rocks and its significance in provenance studies of sedimentary rocks: Journal of Sedimentary Petrology, 33, 559-579.

Blatt, H., Middleton G., Murray, R., 1972, Origin of sedimentary rocks: Englewood Cliffs, New Jersey, Prentice-Hall, 634 p.

Buffler, R.T., Sawyer, D.S., 1985, Distribution of crust and early history, Gulf of Mexico Basin: Gulf Coast Association of Geological Societies Transactions, 35, 333-334.

Cameron, K.L., Blatt, H., 1971, Durabilities of sand size schist and "volcanic" rock fragments during fluvial transport, Elk Creek, Black Hills, South Dakota: Journal of Sedimentary Petrology, 41, 2, 565-576.

Campa, M.F., Coney, P.J., 1983, Tectono-stratigraphic terranes and mineral resource distributions in Mexico: Canadian Journal Earth Sciences, 20, 1040-1051.

Carrillo-Bravo, J., 1961, Geología del Anticlinorio Huizachal-Peregrina al NW de Ciudad Victoria, Tampaulipas: Boletín de la Asociación Mexicana de Geólogos Petroleros, 13, 1-98.

Cox, R., Lowe, D.R., 1995, A conceptual review of regional-scale controls on the composition of clastic sediments and the co-evolution of continental blocks and their sedimentary cover: Journal of Sedimentary Research, 65 (1a), 1-12.

Critelli, S., 1999, The interplay of lithosferic flexure and thrust accommodation in forming stratigraphic sequences in the southern Apennines foreland basin system, Italy: Rendiconti Lincei, 10, 257-326.

Critelli, S., Ingersoll, R.V., 1994, Sandstone petrology and provenance of the Siwalik Group (northwestern Pakistan and western-southeastern Nepal): Journal of Sedimentary Research, 64 (4a), 815-823.

Critelli, S., Le Pera, E., 1995, Tectonic evolution of the Southern Apennines Thrust-Belt (Italy) as Reflected in Modal Compositions of Cenozoic Sandstone: The Journal of Geology, 103, 95-105.

Critelli, S., Marsaglia, K.M., Busby, C.J., 2002, Tectonic history of a Jurassic backarc-basin sequence (the Gran Cañon Formation, Cedros Island, Mexico), based on compositional modes of tuffaceous deposits: Geological Society of America Bulletin, 114, 515-527.

Critelli, S., Nilsen, T.H., 2000, Provenance and stratigraphy of the Eocene Tejon Formation, Western Tehachapi Mountains, San Emigdio Mountains, and Southern San Joaquin Basin, California: Sedimentary Geology, 136, 7-27.

Decelles, P.G., Hertel, F., 1989, Petrology of fluvial sands from the Amazonian foreland basin, Peru and Bolivia: Geological Society of America Bulletin, 101, 1552-1562.

Díaz-Gonzalez,T., 1953, Itinerario de la excursión al Cañón de la Peregrina, Tamaulipas, en Primera Convención: México D.F., Asociación Mexicana de Geólogos Petroleros, Libro Guía, 6 p.

Dickinson, W.R., 1970, Interpreting detrital modes of graywacke and arkose: Journal of Sedimentary Petrology, 40, 695-707.

Dickinson, W.R., 1985, Interpreting provenance relations from detrital modes of sandstones, en Zuffa, G.G. (ed), Provenance of Arenites: Dordrech, The Netherlands, D. Reidel Publishing Company, 333362 .

Dickinson, W.R., Beard, L.S., Brakenridge, G.R., Erjavec, J.L., Ferguson, R.C., Inman, K.F., Knepp, R.A., Lindberg, F.A., Ryberg, P.T., 1983, Provenance of North American Phanerozoic sandstones in relation to tectonic setting: Geological Society of America Bulletin, 94, 222-235.

Dickinson, W.R., Suczek, C.A, 1979, Plate tectonics and Sandstone Compositions: The American Association of Petroleum Geologists Bulletin, 63, 2164-2182.

Dowe, D.S., Nance, R.D., Keppie, J.D., Cameron, K.L., Ortega-Rivera, A., Ortega-Gutierrez, F., Lee, J.W.K., 2005, Deformational history of the Granjeno Schist, Ciudad Victoria, México: Constraints on the closure of the Rheic Ocean?: International Geology Review, 47, 920-937.

Eguiluz de Antuñano, S., 1990, La formación Carbonera y sus Implicaciones Tectónicas, Estados de Coahuila y Nuevo León: Boletín de la Sociedad Geológica Mexicana, 51,1-37. 
Eguiluz de Antuñano, S., 2001, Geologic evolution and gas resources of the Sabinas Basin in Northeastern Mexico, en Bartolini, C., Buffler, R.T., Cantú-Chapa, A., (eds), The Western Gulf of Mexico Basin: Tectonics, Sedimentary Basins, and Petroleum Systems: Tulsa, Oklahoma, The American Association of Petroleum Geologists, Memoir 75, 241-270.

Fastovsky, D.E., Don Hermes, O., Strater, N.H., Bowring S.A., Clark, J.M., Montellano, M., Hernandez, R.R., 2005, Pre-Late Jurassic fossilbearing volcanic and sedimentary red beds of Huizachal Canyon, Tamaulipas, Mexico, en Anderson T.H., Nourse J.,A, McKee J. W., Steiner M.B. (eds.), The Mojave-Sonora Megashear Hypothesis: development, assessment, and alternatives, Geological Society of America, Special Paper 393, 401-426.

Fillon, R.H., 2007, Mesozoic Gulf of Mexico basin evolution from a planetary perspective and petroleum system implications: Petroleum Geoscience, 13, 105-126.

Garzanti, E., Andò, S., Vezzoli, G., Dell'Era, D., 2003, From rifted margins to foreland basins: Investigating provenance and sediment dispersal across desert Arabia (Oman, U.A.E.): Journal of Sedimentary Research, 73, 572-588.

Garzanti, E., Vezzoli, G., 2003, A classification of metamorphic grains in sands based on their composition and grade: Journal of Sedimentary Research, 73, 830-837.

Garzanti, E., Vezzoli, G., Andò, S., Castiglioni, G., 2001, Petrology of Rifted-Margin Sand (Red Sea and Gulf of Aden, Yemen): Journal of Geology, 109, 277-297.

Goldhammer, R.K., 1999, Mesozoic sequence stratigraphy and paleogeographic evolution of northeast Mexico, en Bartolini, C., Wilson, J.L., Lawton, T.F. (eds), Mesozoic sedimentary and tectonic history of north-central Mexico: Boulder, Colorado, Geological Society of America, Special Paper 340, 1-58.

Goldhammer, R.K., Johnson, C.A., 2001, Middle Jurassic-Upper Cretaceous paleogeographic evolution and sequence-stratigraphic framework of the northwest Gulf of Mexico rim, en Bartolini, C., Buffler, R.T., Cantú-Chapa, A. (eds), The Western Gulf of Mexico Basin: Tectonics, Sedimentary Basins, and Petroleum Systems: Tulsa, Oklahoma, The American Association of Petroleum Geologists, Memoir 75, 45-81.

González-Acebrón, L., Arribas, J., Mas, R., 2007, Provenance of fluvial sandstones at the start of late Jurassic-Early Cretaceous rifting in the Cameros Basin (N. Spain): Sedimentary Geology, 202,138-157.

Götte, M., 1988, Estudio geológico y estructural de Galeana N.L. (México) y sus alrededores: Linares, Nuevo León, México, Universidad Nacional Autónoma de Nuevo León, Actas de la Facultad de Ciencias Tierra, 3, 61-87.

Grajales-Nishimura, N., Terrell, D.J., Damon, P.E., 1992, Evidencias de la prolongación del arco magmático cordillerano del Triásico Tardío-Jurásico en Chihuahua, Durango y Coahuila: Boletín de la Asociación Mexicana de Geólogos Petroleros, 42, 1-18.

Gursky, H.J., Michalzik, D., 1989, Lower Permian Turbidites in the Northern Sierra Madre Oriental: Zentralblatt für Geologie und Paläontologie, Geology Paleontology, 1, 821-838.

Hiscott, R.N., 1978, Provenance of Ordovician deep-water sandstones, Tourelle Formation, Quebec, and implications for initiation of the Taconic orogeny: Canadian Journal Earth Science, 15, 1579-1597.

Imlay, R.W., 1938, Ammonites of the Taraises Formation of northern Mexico: Geological Society of America Bulletin, 49, 539-602.

Ingersoll, R., 1983, Petrofacies and provenance of Late Mesozoic Forearc Basin, Northern and Central California: The American Association of Petroleum Geologists Bulletin, 67, 1125-1142.

Ingersoll, R.V., Bullard, T.F., Ford, R.L., Grimm, J.P., Pickle, J.D., Sares, S.W., 1984, The effect of grain size on detrital modes; a test of the Gazzi-Dickinson point-counting method: Journal of Sedimentary Petrology, 54, 103-116.

Jenchen, U., 2001, Fazies und Geochemie in kontinentalen TriasBecken im westlichen Argentinien und in Patagonien $\left(30^{\circ}-50^{\circ} \mathrm{S}\right)$ : Münter, Alemania, Münstersche Forschungen zur Geologie und Paläontologie, $91 \mathrm{p}$.
Jenchen, U., 2007a, Análisis sedimentológico, petrográfico y geoquímico de sedimentos clásticos en el Noreste de México, Proyecto CT 1377-06: Linares, Nuevo León, México, Universidad Aautónoma de Nuevo León, Programa de Apoyo a la Investigación Científica y Tecnología, Reporte técnico anual (inédito), $24 \mathrm{p}$.

Jenchen, U., 2007b, La Popa Basin, NE Mexico: an Analog for Near Salt Deformation and Hydrocarbon Trapping: Linares, Nuevo León, México, Facultad de Ciencias de la Tierra, Universidad Autónoma de Nuevo León, Force-Field Trip to the La Popa Basin, libro guía, 60 p.

Johnson, M.J., 1993, The system controlling the composition of clastic sediments, en Johnson, M.J., Basu, A. (eds), Processes controlling the composition of clastic sediments: Boulder, Colorado, Geological Society of America, Special Paper 284, 1-19.

Jones, N.W., Mckee, J.W., Anderson, T.H., Silver, L.T., 1995, Jurassic volcanic rocks in northeastern Mexico: A possible remnant of a Cordilleran magmatic arc, en Jackes-Ayala, C., Gonzáles-León, C.M., Roldán-Quintanilla, J. (eds.), Studies on the Mesozoic of Sonora and adjacent areas: Boulder, Colorado, Geological Society of America, Special Paper 301, 179-190.

Keppie J.D., 2004, Terranes of Mexico Revisited: A 1.3 Billion year Odyssey: International Geology Review, 46, 765-794.

Mack, G.H., 1984, Exceptions to the relationship between plate tectonics and sandstone composition: Journal of Sedimentary Petrology, 54, 212-220.

Marsaglia, K.M., 1991, Provenance of sand and sandstones from a rifted continental arc, Gulf of California, Mexico, en Fisher R.V., Smith G.A., (eds), Sedimentation in Volcanic Settings: Society for Economic Paleontologists and Mineralogist, Special Publication $45,237-248$.

Makowitz, A. Sibley, D., 2001, Crystal Growth Mechanisms of Quartz Overgrowths in a Cambrian Quartz Arenite: Journal of Sedimentary Research, 71, 809-816.

Marsaglia, K.M., Ingersoll, R.V., Packer, B.M., 1992, Tectonic evolution of the Japanese islands as reflected in modal compositions of Cenozoic forearc and backarc sand and sandstone: Tectonics, 11, 1028-1044.

Marsaglia, K.M., Pavia, J.A., Maloney, S.J., 2007, Petrology and provenance of Eocene-Albian sandstones and grainstones recovered during ODP Leg 210: implications for passive margin (rift-to-drift) sandstone provenance models, en Tucholke, B.E., Sibuet, J.C., Klaus, A. (eds.), Proceedings of Ocean Drilling Program: Texas, College Station, Ocean Drilling Program, Scientific Results 210, 1-47.

Marsaglia, K.M., Tazaki, K., 1992, Diagenetic trends in ODP Leg 126 sandstones, en Taylor, B., Fijioka, K. (eds.), Proceedings of the Ocean Drilling Program: Texas, College Station, Ocean Drilling Program, Scientific Results 126, 125-138.

McBride, E.F., 1963, A classification of common sandstones: Journal of Sedimentary Petrology, 33, 664-669.

McKee, J.W., Jones, N.W., Long, L.E., 1990, Stratigraphy and provenance of strata along the San Marcos fault, central Coahuila, Mexico: Geological Society of America Bulletin, 102, 593-614.

Meiburg, P., Chapa-Guerrero, J.R., Grotehusmann, I., Kustusch, T., Lentzy, P., de León-Gómez, H., Mansilla-Teran, M.A., 1987, El basamento PreCretácico de Aramberrí: estructura clave para comprender el décollement de la cubierta Jurásica / Cretácica de la Sierra Madre Oriental, México: Linares, Nuevo León, México, Universidad Autónoma de Nuevo León, Actas Facultad de Ciencia de la Tierra, 2, 15-22.

Michalzik, D., 1986, Stratigraphy and Paleogeographic of the Northeast Sierra Madre Oriental, Mexico (Triassic-Lowermost Cretaceous): Zentralblatt für Geologie und Paläontologie, Geology Paleontology, 1, 11-19.

Michalzik, D., 1988, Trias bis tiefste Unter-Kreide der nordöslichen Sierra Madre Oriental, Mexico. Fazielle Entwicklung eines passiven Kontinentalrandes: Darmstad, Alemania, tesis doctoral, 247 p.

Michalzik, D., 1991, Facies sequence of Triassic-Jurrasic red beds in the Sierra Madre Oriental (NE Mexico) and its relations to the early opening of the Gulf of Mexico: Sedimentary Geology, 71, 243-259. 
Michalzik, D., Shumann, D., 1994, Lithofacies relations and palaecology of a Late Jurassic to Early Cretaceous fan delta to shelf depositional system in the Sierra Madre Oriental of north-east Mexico: Sedimentology, 41, 463-477.

Mickus, K., Stern, R.J., Keller, G.R. Anthony, E.Y., 2009, Potential field evidence for a volcanic rifted margin along the Texas Gulf Coast: Geology, 37, 387-390.

Mixon, R.B., Murray, G.E., Díaz, T., 1959, Age and correlation of Huizachal Group (Mesozoic), state of Tamaulipas, Mexico: American Association of Petroleum Geologists Bulletin, 43, 757 771.

Nesbitt, H.W., Fedo, C.M., Young, G.M., 1997, Quartz and Feldspar Stability Steady, and Non-steady-State Weathering, and Petrogenesis of Siliciclastic Sands and Muds: The Journal of Geology, 105, 173-192.

Nilsen, T.H. Sylvester, A.G., 1995, Strike-slip basins, en Busby, C.J., Ingersoll, R.V. (eds): Tectonics of Sedimentary Basins: Cambridge, UK, Blackwell, 425-457.

Nilsen, T.H., Sylvester, A.G., 1999, Strike-slip basins: Part 1: The Leading Edge, 18, 1146-1152.

Ocampo-Díaz Y.Z.E., Jenchen U., Guerrero-Suástegui, M., 2008, Facies y sistemas de depósito del Miembro Arenoso Galeana (Formación Taraises, Cretácico Inferior, NE de México): Revista Mexicana de Ciencias Geológicas, 25, 438-464

Ocampo-Díaz, Y.Z.E., 2007, Evolución sedimentológica y geoquímica del Miembro Arenoso Galeana (Formación Taraises): Linares, Nuevo León, México, Universidad Autónoma de Nuevo León, Facultad de Ciencias de la Tierra, tesis de maestría, $276 \mathrm{p}$.

Ocampo-Díaz, Y.Z.E., 2011, Implicaciones tectono-sedimentarias de las intercalaciones clásticas en el límite Jurásico-Cretácico del noreste de México (Fosa de Monterrey y Cuenca de Sabinas): Linares, Nuevo León, México, Universidad Autónoma de Nuevo León, Facultad de Ciencias de la Tierra, tesis doctoral, $275 \mathrm{p}$.

Parrado Román, J.M., Achab, M., 1999, Grain-size trends associated with sediment transport patterns in Cadiz Bay (southwest Iberian Peninsula): Boletín del Instituto Español de Oceanografía, 15 269-282.

Pettijohn, F.J., Potter, P.E., Siever, R., 1972, Sand and Sandstone: New York, Springer-Verlag, $553 \mathrm{p}$.

Pindell J., Kennan L., 2002, Exploration Framework Atlas Series: Volume 4: Mexico and Gulf of Mexico: Londres, Tectonics Analysis, Ltd., 200 p.

Pittman, E.D., 1969, Destruction of Plagioclase twins by stream transport: Journal Sedimentary Petrology, 39, 1432-1437.

Prosser, S., 1993, Rift-related linked depositional system and their seismic expression, in Williams, G.D., Dobb, A. (eds.), Tectonics and Seismic Sequence Stratigraphy: London, Geological Society of London, Special Publication 71, 35-66.

Reading, H.G., 1980, Characteristics and Recognition of Strike-Slip Fault Systems, en Ballance, P.F., Reading, H.G., (Eds.), Sedimentation in Oblique-Slip Mobile Zones: The International Association of Sedimentologits, Blackwell Publishing Ltd., London, U.K. Special Publication 4, 7-26.
Ricci-Lucchi, F. 1985, Influence of transport processes and basin geometry on sand composition, en Zuffa, G.G. (ed.), Provenance of Arenites: Dordrecht, Países Bajos, D. Reidel Publishing Company, 309-332.

Rubio-Cisneros I.I., Lawton, T.F, 2011, Detrital zircon U-Pb ages of sandstones in continental red beds at Valle de Huizachal, Tamaulipas, NE Mexico: Record of Early-Middle Jurassic arc volcanism and transition to crustal extension: Geosphere, 7, 159-170

Salvador A., 1991, Origin and development of the Gulf of Mexico basin, en Salvador A. (ed.), The Geology of North America: The Gulf of Mexico Basin: Boulder, Colorado, The Geological Society of America, J, 389-444.

Spencer, D.W., 1963, The interpretation of grain size distribution curves of clastic sediments.: Journal of Sedimentary Research., 33, 180-190.

Suttner, L.J., Basu, A., Mack, G.H., 1981, Climate and the origin of quartz arenites: Journal of Sedimentary Petrology, 51, 1235-1246.

Sylvester, A.G., 1988, Strike-slip faults: Geological Society of America Bulletin, 100, 1666-1703.

Tardy, M., Blanchet, R., Zimmermann, M., 1989, Les linéaments du Texas et Caltam entre Cordilléres Américanes et Sierras Madre Mexicanes: Nature, Origine et Évolution structurale: Bulletin des Centre de Recherches Exploration-Production Elf-Aquitaine, 13, 219-227.

Torres, R., Ruiz, J., Patchett, P.J., Grajales J.M., 1999, Permo-Triassic continental arc in eastern Mexico: Tectonic implications for reconstructions of southern North America, en Bartolini, C., Wilson, J.L., Lawton, T.F. (eds.), Mesozoic sedimentary and tectonic history of north-central Mexico: Boulder, Colorado, Geological Society of America, Special Paper 340, 191-196.

Tortosa, A., Palomares, M., Arribas, J., 1991, Quartz grain types in Holocene deposits from the Spanish Central System: some problems in provenance analysis, en Morton, A.C., Todd, S.P., Haughton, P.D.W. (eds.), Developments in Sedimentary Provenance Studies: London, Geological Society, Special Publication 57, 47-54.

Valloni, R., 1985, Reading provenance from modern sands, en Zuffa, G.G. (ed.), Provenance of Arenites: Dordrecht, Países Bajos, D. Reidel Publishing Company, 309-332.

Visher, G.S., 1969, Grain size distributions and depositional processes: Journal of Sedimentary Petrology, 39, 3, 1074-1106.

Young, S.W., 1976, Petrographic textures of detrital polycristalline quartz as an aid to interpreting crystalline source rocks: Journal of Sedimentary Petrology, 46, 595-603.

Zuffa, G.G., Gaudio, W., Rovito, S., 1980, Detrital mode evolution of the rifted continental-margin Longobucco sequence (Jurassic), Calabrian Arc, Italy: Journal of Sedimentary Petrology, 50, 51-61.

Manuscrito recibido: Mayo 16, 2010.

Manuscrito corregido recibido: Marzo 26, 2011.

Manuscrito aceptado: Abril 23, 2011. 